\title{
5 "Die machen uns die Moleküle" - Zur Grenzarbeit zwischen Biomedizin und Chemie
}

Das Verhältnis von chemischen Wissenschaften und den Feldern der Biomedizin ist seit der Entwicklung der akademischen Chemie im frühen 19. Jahrhundert als medizinische Hilfswissenschaft durch einen Modus der Arbeitsteilung geprägt. In diesem Sinne haben sich die Felder der Medizin und der Chemie in einem ambivalenten Verhältnis gegenseitiger Abgrenzung und Abhängigkeit konstituiert (Powers, 2012, S. 5f.). Obwohl die Medizin sich im Laufe ihrer Geschichte verstärkt den Naturwissenschaften zugewandt und dadurch an Evidenz und Behandlungserfolgen hinzugewonnen hat, wurde dennoch stets eine inhärente $\mathrm{Ab}$ grenzung in der disziplinären Organisation des Wissens aufrechterhalten. Die Frage danach, wie viel und welche Art von Wissen Mediziner/innen selbst von naturwissenschaftlichen Inhalten benötigen, wird in diesem Zusammenhang immer wieder neu ausgehandelt (ebd., S. 1of.). Aber auch für die Chemie war die Grenzziehung zu den Feldern der Medizin folgenreich und durch Ambivalenzen gekennzeichnet: Auf der einen Seite erlangte die Chemie durch die zahlreichen Anbindungen an medizinische Anwendungen in Analytik, Pharmakologie und Labortechnik ein erhebliches Maß an fachlicher Legitimation, auf der anderen Seite fiel es der Chemie jedoch schwer, sich als eigenständige Disziplin zu behaupten und vom Status einer Hilfswissenschaft zu emanzipieren (Eckart, 2017, S. 191). Diese Arbeitsteilung wurde in der Folge durch forschungspolitische Dispositive der Unterscheidung zwischen grundlagen- und anwendungsorientierter Forschung verankert und reifiziert. Die Genealogie dieser Präidee der Arbeitsteilung wird in Abschnitt 5.1 nachgezeichnet.

Bei der Medizin handelt es sich um ein fluides interdisziplinäres Gefüge aus gesundheitsbezogenen Fachbereichen in den Sozial- und Naturwissenschaften, mit zahlreichen Anbindungen an ethische, rechtliche, ökonomische, soziale und politische Fragen. Mediziner/innen in Behandlung und Forschung zeigen sich stets darum bemüht, ihren eigenen Platz in interdisziplinären Gefügen zu behaupten und die Grenzen ihrer Zuständigkeit zu ziehen (ebd., S. 241ff.). Die Präidee der Arbeitsteilung, die dieser Epistemik und Praxis zugrunde liegt, ist dabei politisch umkämpft. Während die Präidee der Arbeitsteilung Ärzt/innen 
auf der einen Seite ein gewisses Maß an Handlungsfähigkeit ermöglicht, wird sie im Zuge der Innovationsdispositive, die zunehmend auch die Biomedizin in den Blick nehmen, als innovationsfeindlich problematisiert. Dies zeigt sich in den beiden Fallstudien, die in diesem Kapitel diskutiert werden: Erstens in den Diskursen um den Contergan-Fall und der Rolle, die die molekulare Eigenschaft der Chiralität im entsprechenden Thalidomid-Wirkstoff darin einnahm (Abschnitt 5.2), und zweitens dem Programm der translationalen Medizin, das sich in kritischer Abgrenzung zur Präidee der Arbeitsteilung formuliert.

In der Grenzarbeit zwischen Chemie und Medizin stellt der Fall des Arzneimittels Contergan, das in den frühen 1960er Jahren als Massenmedikament vermarktet wurde und aufgrund unzureichender Pharmakovigilanztests körperliche Fehlbildungen bei weltweit 10.000 Neugeborenen verursacht hatte (Kirk, 1999), einen Knotenpunkt dar. Der Contergan-Fall erinnert stets daran, dass die etablierte Arbeitsteilung zwischen Chemie, Pharmazie und Medizin mit dramatischen Folgen versagen kann. So lässt sich in den entsprechenden Narrationen und Rekonstruktionen des Contergan-Falls beobachten, wie es über den Verweis auf Contergan in den Chemiekursen der Mediziner/innen-Ausbildung der Chemie gelingt, die Relevanz ihrer Gegenstände, Konzepte und Modelle in der Medizinausbildung aufrechtzuerhalten. Wie in der Interviewstudie deutlich wird, stellt die Chemie für Mediziner/innen nach wie vor die zentrale Sozialisationsinstanz in die molekulare Welt dar. Die Chemievorlesungen und Praktika im Medizinstudium prägen die Wahrnehmung, Körper- und Krankheitsbilder von angehenden Mediziner/innen noch in der Gegenwart nachhaltig mit. Dennoch erscheinen die chemischen Inhalte im ohnehin völlig überladenen Medizinstudium zunehmend rechtfertigungsbedürftig: Erstens sind insbesondere die Methoden chemischer Analytik fast vollständig aus dem ärztlichen Alltag externalisiert worden und zweitens werden seit der Bio-Molekularisierung der Medizin zunehmend molekularbiologische bzw. biotechnologische Zugriffe auf das Molekulare in der Medizin dominant.

Wie in Abschnitt 5.3 ausgeführt wird, haben sich aus der Hybridisierung von Feldern im Zuge der Bio-Molekularisierung der Medizin neue Probleme ergeben: Wissensbestände, Betrachtungsebenen, disziplinäre Kulturen sind demnach immer weniger untereinander anschlussfähig, was in einem kettenförmigen, linearen Innovationsmodell nahtlos ineinander übergreifender Instanzen zu inhärenten Widersprüchen führt. Das beobachtete Auseinanderdriften von Disziplinen in der Arzneistoffentwicklung und die sich immer stärker ausdifferenzierenden und zuspitzenden Expertisen brachten in der Folge das Translationsparadigma hervor, das ebenfalls von der Pharmaindustrie aus in viele verschiedene Ingenieurswissenschaften übernommen wurde (Chai und Shih, 2013, S. 3). Das Konzept der translationalen Medizin (TM) etwa bedient sich dessen Grundannahmen, indem die geringe Geschwindigkeit von Innovationsprozessen kritisch auf mangelhafte 
Wissensübersetzungen zwischen verschiedenen Disziplinen und Instanzen zurückgeführt wird (Zerhouni, 2005). Dieser zweite Beispieldiskurs zeigt ebenfalls, dass die Chemie zugunsten der hybriden Lebenswissenschaften an Einfluss auf die medizinische Vorstellung von der molekularen Welt einbüßt.

\subsection{Die Präidee der Arbeitsteilung zwischen Chemie und Biomedizin}

Die Medizin präsentiert sich heute als komplexes, interdisziplinäres Feld verschiedener Lebens- und Gesundheitswissenschaften sowie Technosciences, die eine breite Palette von Forschung, Patient/innen-Versorgung und populationsbezogenem Gesundheitsmanagement umfassen und über Anbindungen an die Sozialund Naturwissenschaften verfügen (Egloff et al., 2011). Vor diesem vielschichtigen Hintergrund erscheint die Medizin wie ein Knotenpunkt im sich kontinuierlich wandelnden, disziplinären Gefüge der Wissenschaften vor dem Hintergrund politischer, sozialer, ökonomischer und technischer Verwobenheiten. In ihrer Rolle als Anwendungsgebiet haben Medizin und Gesundheit durch die Geschichte hinweg unterschiedlichen disziplinären Kollektiven in der Akquirierung von Ressourcen als Legitimation gedient: das Versprechen, dass chemisches, biophysikalisches, psychologisches oder literaturwissenschaftliches ${ }^{1}$ Wissen einen Nutzen für kranke Menschen hat, stellt ein intelligibles Narrativ dar. Auf diesem Wege wurden medizinische Menschen-, Körper- und Krankheitsbilder (in der Folge als Präideen bezeichnet) im historischen Verlauf durch zahlreiche feld- und disziplinspezifische Narrative, Projektionen, theoretische Prämissen, Innovationsdispositive und technologische Bedingungen des Sehens geprägt (Fleck, 2014a). Aber auch umgekehrt beeinflusste die Medizin mit ihrem sich kontinuierlich wandelnden ärztlichen Blick, ihren Problemstellungen, Entdeckungen, Moralvorstellungen und Katastrophen die Nachbarwissenschaften. Das Feld der Medizin bietet sich aus diesem Grunde in besonderen Maße für das Studium disziplinärer Dynamiken und insbesondere ihrer Abgrenzungsbestrebungen - sei es gegen außerwissenschaftliche Kreise oder in Form interdisziplinärer Grenzarbeit - an.

Die Analyse der Präideen der gegenwärtigen Biomedizin offenbart eine Genealogie der Grenzarbeit zwischen biologischen und chemischen Disziplinen in ihrem Zugriff auf die molekulare Welt, die sich als umkämpfte Arena präsentiert. terung der klassischen Disziplinen der Geschichte, Theorie und Ethik der Medizin. Von der Einbindung weiterer Disziplinen der Sozial- und Ceisteswissenschaften sowie der Künste versprechen sich ihre Vertreter/innen Zugewinne für den Medizinbetrieb, da diese Perspektiven soziale und kulturelle Aspekte von Cesundheit und Krankheit in die medizinische Wissensproduktion integrieren, die Krankheitsverläufe und Behandlungserfolge ebenso bedingen wie biologische oder Umweltfaktoren (Biller-Andorno and Roduit, 2016). 
Die Menschen-, Körper- und Krankheitsbilder der Medizin sind gegenwärtig im Wesentlichen durch diejenigen der molekularbiologischen und -technologischen Life Sciences geprägt, die sich aus der Biologie heraus entwickelt haben (Löwy, 2011, S. 117). Allerdings, so zeigen die Analysen dem empirischen Materials, ist der ärztliche Zugriff auf die molekulare Welt nach wie vor durch einen organischchemischen style of reasoning geprägt und die neueren, biotechnologischen Zugriffe diesen nachgeordnet. Die Wissensbestände der Chemie, die im 19. Jahrhundert erheblich Einfluss auf medizinische Behandlungsmethoden und Wissensproduktion genommen haben ${ }^{2}$, sind in der Selbstwahrnehmung der medizinischen Felder selbst sowie auch in der Medizingeschichte in Vergessenheit geraten. Vielmehr, so zeigt die empirische Untersuchung, gilt ein chemischer Zugriff auf die Medizin und das Molekül als veraltet und dem biotechnologischen gegenüber als unterlegen. Diese These wird zunächst durch die historische Betrachtung dieses Abschnitts gestützt, die sich kritisch mit den kanonisierten Narrativen der Medizingeschichte auseinandersetzt, insbesondere mit dem Konzept der Molekularisierung der Medizin und dem Begriff der Biomedizin (Löwy, 2011). Die Feststellung lautet, dass insbesondere in der Historiografie zur Molekularisierung der Medizin i.d.R. ausschließlich von der Bio-Molekularisierung der Medizin nach dem Zweiten Weltkrieg die Rede ist, während die Molekularisierung der Medizin durch die Anbindungen an die organische Chemie im 19. Jahrhundert nur marginal berücksichtigt wird. Auch dies ist ein Symptom dafür, dass die organische Chemie in der sozialen Arena des Molekularen zunehmend angesichts disziplinärer Differenzierungen und Hybridisierungen an Bedeutung einbüßt.

Die Präideen der Medizin werden gegenwärtig in der Wissenschaftsforschung primär in ihrer naturwissenschaftlichen, (molekular-)biologischen und biotechnologischen Prägung ${ }^{3}$ wahrgenommen und es fällt schwer, Phänomene von Gesundheit und Krankheit des Körpers jenseits einer (molekular-)biologischen Be-

Als Beispiel für eine enge Verbindung zwischen Medizin und chemischer Analytik sei hier die Balneologie bzw. Kurmedizin des 19. und 20. Jahrhunderts erwähnt. Die Aufklärung der chemischen Zusammensetzung von Wässern und eine entsprechende Zuschreibung von kurativen Eigenschaften galt als besonders intelligibel und war für die Etablierung chemischer Lehrstühle von Bedeutung (Weisz, 2001).

3 Ein lebenswissenschaftlicher Zugriff auf den menschlichen Körper war nicht immer dominant: Etwa die Säftelehre der Humoralpathologie, die seit der Antike bis in die Neuzeit das medizinische Denken prägte, vertrat ein mechanistisches Körperbild (Fleck, 2014b, S. 189) und ist heute im medizinischen Denken weitestgehend in Vergessenheit geraten. Auch die mikroskopische Seite des Lebens und damit in Verbindung stehende Erkrankungen waren lange Zeit nicht durch biologische Wissensproduktion und Weltbilder definiert, sondern u.a. durch die Anbindungen an die Chemie (Eckart, 2017, S. 9off.). So wurde etwa die Welt der Mikroorganismen von (Bio-)Chemiker/innen wie Louis Pasteur erschlossen, die sich als Chemiker/innen an medizinischen Fakultäten bewegten, ehe die mikroskopische Welt der Mikroorganismen als Teil der Biologie verstanden wurde (Latour, 1990). 
grifflichkeit zu denken (Clarke und Shim, 2011). Auch kann der „naturwissenschaftliche, molekular-biologische und biotechnologische" Denkstil kaum als einheitlich bezeichnet werden, sondern präsentiert sich angesichts der Hybridisierung der Lebenswissenschaften in zahlreiche Teilbereiche sowie delokalisierte Orte und Modi der Wissensproduktion als vielstimmiges und unscharfes Feld (Egloff et al., 2011, S. 9). Dies zeigt sich etwa in den Assemblagen, die einzelne Krankheitsbilder im klinischen Kontext darstellen, wo sehr viele Perspektiven, Epistemologien und Methoden aufeinander treffen (Mol, 2003). In diesem polyphonen Konglomerat bestehen verschiedene Modellvorstellungen des „molekular charakterisierten Menschen“ (wie auch der molekularen Welt) nebeneinander (ebd., S. 13). Der Mensch als „Modell seiner kleinsten Teile“ erfährt angesichts der Pluralität und Hybridität disziplinärer Zugriffe eine Auflösung in Betrachtungsebenen und medizinische Phänomene verwachsen sich $\mathrm{zu}$ vielschichtigen Assemblagen und translationalen Objekten an den Schnittstellen verschiedener Denkkollektive (Michael, 2011, S. 65). Die Auflösung des Körpers in verschiedene molare und molekulare Grenzobjekte und Perspektiven erfolgte in drei Phasen, die in der Folge geschildert werden: die chemische Molekularisierung, die molekularbiologische Molekularisierung und die biotechnologische Molekularisierung.

Wie in Kapitel 2.1 diskutiert wird, entwickelte sich die Chemie im 18. Jahrhundert zunächst als Hilfswissenschaft der Medizin (neben dem Minen- und Hüttenwesen sowie der Geologie) an europäischen Universitäten und erlangte erst dank der Legitimation durch die großindustriellen Erfolge Mitte des 19. Jahrhunderts volle Autonomie als wissenschaftliche Disziplin, als europaweit erstmals hunderte, in Vollzeit entlohnte, professionell ausgebildete Chemiker ${ }^{4}$ beschäftigt waren (Bensaude-Vincent und Stengers, 1996, S. 96). Die Chemie etablierte sich zudem mit eigenen, national organisierten Fachgesellschaften, Publikationsorganen, Lehrbüchern, Lehrstätten und - als erste Disziplin überhaupt - mit internationalen Fachkongressen (ebd.). Dennoch befanden sich die chemischen Felder insbesondere zur Zeit ihrer Herauslösung aus dem Status einer Hilfswissenschaft in einem vielschichtigen Abgrenzungsverhältnis. Einerseits herrschte dabei Konkurrenz unter den lokalen wie nationalen chemischen Institutionen und Ausbildungsplänen. Andererseits entstand ein Schisma zwischen der sog. reinen und angewandten Chemie im Kontext der rasch fortschreitenden Industrialisierung erheblichem Maße auf außeruniversitäre Förderung seiner Forschung und Lehre angewiesen (Brock, 1999, S. 8). Gleiches gilt für die frühen, unabhängigen Institute und Laboratorien, an denen seit den 1840er Jahren chemische Forschung und Ausbildung nicht mehr alleinig im Dienste der Apothekerausbildung sowie geologischer, metallurgischer, botanischer, zoologischer oder pharmazeutischer Wissenschaften stand, sondern eigene Forschungsziele und -Interessen verfolgen konnten (Weyer, 2018b, S. 227f.). 
des Faches (ebd., S. 102). Konstant blieb dabei der Bezug auf medizinische Anwendungen und die Ausbildung angehender Mediziner/innen, sodass die Chemie sich von einer sekundär gesetzten Hilfswissenschaft zu einer unentbehrlichen Grundlagenwissenschaft und Sozialisationsinstanz transformierte.

Noch heute stellt die organische Chemie die zentrale Sozialisationsinstanz angehender Mitglieder des Medizinbetriebs dar und dient als gatekeeper für die molekulare Welt. Student/innen der Human-, Zahn- und Veterinärmedizin erhalten eine verpflichtende Ausbildung in den grundständigen Methoden, Konzepten und Theorien der Physik, Chemie und Biologie, deren Relevanz sich daraus begründet, dass Mediziner/innen diese Prinzipien verstehen müssen, um medizinischen Nutzen für Patient/innen daraus ziehen zu können. Im Bereich der Chemie besteht der Lehrstoff dabei neben Überblicksvorlesungen zu den chemischen Feldern (mit Schwerpunkten in organischer Stereochemie und Pharmakologie) aus einem ausführlichen Laborpraktikum. I.d.R. werden diese Lehrpraktika so organisiert, dass externe Lehraufträge an chemische Institute derselben Hochschule vergeben werden, an denen spezielle Schullabore eingerichtet und Vorlesungen mit Titeln wie „Allgemeine Chemie für Mediziner“ entworfen werden. Die organischen Strukturmodelle dienen dabei wie bei angehenden Chemiker/innen auch als Medien des molekularen tacit knowledge, denn die chemische Symbolsprache von Strukturmodellen, Summenformeln und Reaktionsgleichungen zu beherrschen, ist bislang der einzige Weg, einen Zugang zur molekularen Welt zu erlangen (Nesper, 2001). Auf diesem Wege wird auch die Arbeitsteilung zwischen Medizin und Chemie (bzw. den Naturwissenschaften im Allgemeinen) in der Sozialisation ins Feld als Präidee eingeübt und in der Fachidentität verankert.

Die Chemie behielt sich ein ambivalentes Verhältnis zur Medizin bei: Einerseits gelang es ihr, sich aus dem Status einer reinen Hilfswissenschaft zu emanzipieren, da durch die Entwicklung der molekularen Strukturchemie ein eigener epistemischer Raum geschaffen wurde, der eine eigenständige, genuin chemische, Auseinandersetzung mit der mikroskopischen und makroskopischen Natur erlaubte. Andererseits brach die Beziehung zur Medizin nie völlig ab, sondern wurde stets strategisch in der Akquisition von Ressourcen aufrechterhalten. Die Chemie prägte vom 19. bis ca. zur Mitte des 20. Jahrhunderts die Denkstile, Präideen und mentalen Modelle der Medizin nachhaltig mit (Boyer, 2011, S. 188). Chemische Methoden der Destillation, Kristallisation und Analytik gehörten in diesem Zeitraum zur Grundausstattung jeden Krankenhauses, wie Ludwik Flecks zeitgenössische Analysen zur Entstehung wissenschaftlicher Tatsachen eindrücklich belegen (Fleck, 2012, S. 71f.). Die Einübung des ärztlichen Blicks in der Patient/innen-Versorgung erfolgte in Auseinandersetzung mit beiderlei: Einerseits dem kranken molaren Körper und seiner makroskopisch wahrnehmbaren Symptome und andererseits der serologischen Analyse auf der Suche nach krankheitsverursachenden Mikroorganismen, toxischen Stoffen oder körperlichen Ab- 
wehrmechanismen (Fleck, 2014b). In diesem Sinne entwickelte sich die Medizin zu einer Laborwissenschaft vorwiegend durch den Kontakt mit den chemischen Wissenschaften (Rosenfeld, 2002). Biochemische Prozesse wie Antikörperbildung, die Entwicklung von Impfstoffen und die ersten standardisierten Testverfahren auf bestimmte Krankheitsbilder wurden als explizit chemische Methoden und Tätigkeiten betrachtet und die Mikrobiologie speiste sich erst mit der Zeit aus der Chemie heraus (ebd.).

Dennoch gelang der Medizin innerhalb dieser komplexen Boundary Work eine entscheidende Leistung, die das Verhältnis von Medizin und Naturwissenschaften bis heute dominiert: Der eigentlichen Heilerfolg - sei es nun durch verabreichte Badekuren, Tabletten, Gebet, radioaktive Bestrahlung, Massagen oder Aderlass - wird stets als ärztlicher Erfolg verbucht, während die Disziplinen, aus denen sich die vielfältigen Methoden der Medizingeschichte speisten, in dieser Semantik nicht berücksichtigt werden (Folkers, 2011, S. 202). Diese Asymmetrie ist derart im Alltagsverständnis von Medizin und Naturwissenschaft verankert, dass sich etwa chemophobe Narrative bedienen lassen: Während etwa Ärzt/innen im Rahmen der Verschreibung von Chemopharmazeutika der Heilerfolg zugeschrieben wird, werden den eigentlichen Entwickler/innen und Hersteller/innen eher unlautere Interessen unterstellt (ebd.). Das Verhältnis von Medizin und Chemie wird tendenziell eher in der Chemiegeschichte verhandelt und ist dort heute selbst Teil der Grenzarbeit der chemischen Felder, wie sie von diesem noch jungen historischen Zweig geleistet wird (vgl. Abschnitt 5.3). In der Medizingeschichte hingegen wird die Rolle der Chemie kaum berücksichtigt und es herrscht meinen Studien zufolge nach wie vor das Narrativ der Chemie als service science vor. Dies zeigt sich u.a. darin, dass die Molekularisierung der Medizin primär in den Kontext der molekularbiologischen Revolution gesetzt wird:

Die in der Medizingeschichte als eigentliche Molekularisierung der Medizin kanonisierte Entwicklung setzt demnach nicht an den genannten molekularstrukturellen chemischen Einflüssen an, sondern bezeichnenderweise an der Einführung molekularbiologischer Methoden und Konzepte. Die lange Abwesenheit chemischer Perspektiven in der Wissenschaftsforschung (vgl. 5.3) zeigt sich darin ebenfalls wie die Grenzarbeit zwischen chemischen und biowissenschaftlichen bzw. -technologischen Zugriffen auf medizinische Probleme in der sozialen Arena des Molekularen. Die Biologie lieferte zunächst bis zur Mitte des 20. Jahrhundert kaum Weltbilder, Methoden oder Kenntnisse über die molekulare Welt, sondern beschränkte sich ebenso wie die Medizin auf die molare Welt der makro- wie mikroskopischen Organismen (Mittra, 2016a, S. 31). Die Biologie entwickelte sich erst mit ihrer Molekularisierung nach dem Zweiten Weltkrieg flächendeckend von einer Feldwissenschaft (die dem Klassifizieren von Organismen verschrieben war) hin zu einer laborbasierten, experimentellen hard science, die sich an den Kriterien der allgemeinen Wissenschaftskultur mit denen der Physik und Chemie messen 
lassen konnte (ebd.). So unterscheidet sich der Zugriff der klassischen organischen Chemie auf die molekulare Welt von denjenigen der gegenwärtigen Biomedizin, der sich sukzessive hin zu einem hybriden und vielstimmigen Zugriff auf das Molekül entwickelt hat, der die verschiedenen molekularbiologischen und biotechnologischen Disziplinen widerspiegelt, die das Feld medizinischer Forschung gegenwärtig prägen.

Die Molekularisierung der Biologie und der Begriff der Biomedizin wurden in der Medizin- und allgemeinen Wissenschaftshistoriografie als Trends definiert, die $\mathrm{zu}$ den zentralen wissenschaftlichen Entwicklungen des 20 . Jahrhundert gezählt werden. ${ }^{5}$ Die strukturchemischen Wurzeln der Erschließung der molekularen Welt werden in diesen historiografischen Diskursen allerdings wenn überhaupt nur marginal berücksichtigt. Dies wird u.a. darauf zurückgeführt, dass dem intuitiven Verständnis vieler Wissenschaftshistoriker/innen die vitalistische Präidee innewohnt, dass sich chemische Prozesse, die in lebenden Organismen stattfinden, sind grundlegend von denen organischer wie anorganischer Natur unterscheiden müssen, die außerhalb eines solchen ablaufen (Morange, 2012, S. 509f.). Die Verwendung des Begriffs der Molekularisierung fällt in diesen heterogenen Diskursen aber nicht einheitlich aus, sondern ist in seiner Bedeutung diffundiert (Wenkel, 2013, S. 8), was daran liegen mag, da sich unter dem vagen Schirmbegriff der Molekularisierung die verschiedensten historischen Entwicklungen ${ }^{6}$ der Molekularbiologie fassen lassen, was eine sehr weite Begriffsdefinition erlaubt. Auch wenn unter Historiker/innen keine Übereinstimmung darüber herrscht, wann genau die Anfänge und Phasenübergänge der Molekularisierung der Lebenswissenschaften festzulegen sind (Rheinberger, 2008, S. 303), haben sich doch in der Geschichtsschreibung zwei kanonisierte Phasenübergänge dieses Prozesses sedimentiert: Erstens die Transition von einer feldforschenden, auf den molaren Organismus orientierten Biologie hin zu einer molekularen Laborwissenschaft (1940er bis 1960er Jahre) und zweitens die molekulartechnologische Revolution, die das Zeitalter der Lebenswissenschaften als Technosciences in den 1970er Jahren einläutete (ebd. S. 305):

Als analytisches Konzept avancierte die Molekularisierung in der Wissenschaftsgeschichte (aber auch-soziologie, -anthropologie und -philosophie) seit den 1980er Jahren zu einer zentralen Ordungskategorie, die seither ein zentrales Narrativ für die Geschichtsschreibung lebenswissenschaftlicher Disziplinen, Objekte oder Theorien darstellt (Löwy, 2011, S. 120). Da in diesem Bereich sich die Verwobenheiten wissenschaftlicher Paradigmenwechsel mit Wissenschaftspolitik, Privatwirtschaft, Instiutionalisierung, disziplinärer Dynamiken erforschen ließen, prägte dieser Phänomenkomplex die neuere Wissenschaftsforschung der Science und Technology Studies erheblich mit (Filk, 2010, S. 27).

6 Siehe zur Übersicht der als besonders zentral kanonisierten Entwicklungen (Rheinberger, 1995). 
Die sog. molekulare Revolution wird in der Biologie in den 1940er Jahren datiert und fällt den historischen Analysen zufolge in erster Linie mit der Molekularisierung der Genetik und Vererbungslehre zusammen. Die biochemische Forschung hatte hierzu bereits einen Grundstein gelegt und befand sich in Begriff, sich mit der Erforschung von Proteinen, Peptiden und anderen Bio-Molekülen in immer kleinere Bereiche des Lebens vorzuwagen (ebd., S. 305). Die grundlegenden Prozesse des Lebens wurden in der Folge zunehmend anhand mikroskopischer Organismen in vitro erforscht, etwa an Hefezellen, Bakterien und Viren, was zu einer Abwendung von den etablierten makroskopischen Tiermodellen wie Maus und Fruchtfliege führte (ebd.). Darüber hinaus brachten die Forschungsaktivitäten zur DNA-Doppelhelix in den 1950er und die Sequenzierung des menschlichen Genoms in den 1960er Jahren zu einer neuen Metaphorik und Sprache der molekularen Biologie, die insbesondere durch einen neuartigen Informationsbegriff charakterisiert sind (Kay, 2001). Die aufstrebende Molekularbiologie bediente sich in der Folge zahlreichen Analysemethoden und Apparaturen, die aus Chemie und Physik übernommen wurden und die aus Abschnitt 2.3 bekannt sind, zumal einige davon in Auseinandersetzung mit der chiralen Eigenschaft molekularer Strukturen entwickelt worden waren: Chromatografie, Kapillarenelektrophorese, Ultrazentrifuge, NMR und Röntgenstrukturanalyse.

Die Molekularbiologie erwies sich als offen für interdisziplinäre Kooperationen und expandierte in der Folge in andere wissenschaftliche Bereiche (Rheinberger, 2008, S. 305). Auf diese Weise entstand eine molekulare Welt, die sich bezüglich ihrer Methoden, Sprache, symbolischen Repräsentationen, Affiliationen von Forschenden, Orten und Institutionen der Wissensproduktion immer weiter von den Wurzeln in der Chemie ablöste. In den 1970er Jahren zeichnete sich ein weiterer Höhepunkt der Molekularisierung der Lebenswissenschaften ab: Mit der Möglichkeit, die genetischen Codes nicht nur zu beschreiben, zu klassifizieren und abzubilden, sondern mithilfe gentechnologischer Verfahren direkt in diese Prozesse manipulierend einzugreifen, zeichnete sich ein dramatischer Wandel ab (Landecker, 2007). Verschiedene Lebenswissenschaften innerhalb der Landschaft molekularbiologischer Forschung und Entwicklung erfuhren eine Transition hin $\mathrm{zu}$ sog. Technosciences (ebd.). Im Zuge dessen entstand ein weiterer epistemischer Blickwinkel auf das Leben: Neben biochemischen Stoffen und ihren Funktionen in vivo wie in vitro rückten Zelle ${ }^{7}$, Organellen und insbesondere der Zellkern in ihrer molekularbiologischen Verfasstheit ins Zentrum der Aufmerksamkeit (Rose, 2007, S. 65f.). Die Gene werden dabei als molekulare Träger von Information verstanden; sie steuern Vererbung, Reproduktion und die Entwicklung des kollektiven und individuellen Organismus im Sinne der Unterscheidung von Genotechnologischen Objekten wurden siehe (Landecker, 2007). 
und Phänotypen. Die Entschlüsselung des menschlichen Genoms und anderer Spezies sowie die kausale Zuschreibung von genotypischen Ausprägungen und phänotypischen Effekten führte Ende der 1980er Jahre zu einem global angesiedelten mapping project ${ }^{8}$ (Rheinberger, 2006, S. 224).

Die Entwicklung der Molekularbiologie als vage gefasster Großtrend in der zweiten Hälfte des 20. Jahrhunderts ist in erheblichem Maße mit einer Disruption des bestehenden Disziplinengefüges der Naturwissenschaften verbunden. Mit dem Aufkommen des Triple Helix-Innovationsmodells, bei dem akademische Wissenschaft stärker an politische Entscheidungen und industrielle Interessen (insbesondere der pharmazeutischen Industrie) gebunden wurde (Etzkowitz und Leydesdorff, 2000), entstand eine Vielzahl hybrider biowissenschaftlicher und technologischer Disziplinen, die sich aus den drei etablierten Disziplinen der Chemie, Biologie und Physik speisten. Nanobiophysik, Neuro Sciences oder Omics Sciences repräsentieren in ihrer interdisziplinären, anwendungs- und marktbezogenen Ausrichtung dieses neue Paradigma und eine generelle Abwertung der etablierten Großdisziplin (Forman, 1997). Ein gefragter Anwendungsbezug dieser neuen, interdisziplinären Kollektive lag dabei von Beginn an in der Medizin, die ebenfalls in der Folge eine Molekularisierung unterlief und seither als Biomedizin bezeichnet wird (Löwy, 2011). Die Transformation hin zur Biomedizin in Auseinandersetzung mit den entstehenden Life Sciences veränderte das Feld der Medizin auf mehrere Ebenen tiefgreifend: Erstens bezüglich epistemischen Prämissen und Präideen wie dem Körper- und Krankheitsbegriff, zweitens bezüglich des Verhältnisses von Forschung und Praxis und drittens bezüglich einer Arbeitsteilung mit den Naturwissenschaften in der Aushandlung von Verantwortung.

Mit der Entstehung der Biomedizin als Teil der hybriden Landschaft der Lebenswissenschaften erlebte der Krankheitsbegriff der Medizin eine Transition. Der Biomarker, der bereits zur Phase der Chemomedizin biochemisch verfasst war, entsprach dem sog. Schlüssel-Schloss-Modell der Rezeptor-Wirkstoff-Theorie der organischen Chemie des 19. Jahrhunderts (Egloff et al., 2011, S. 13). Als krankhaft beurteilte Körperprozesse konnten im Körper lokalisiert und mithilfe kleiner chemischer Moleküle behoben werden und auch die Diagnostik setzte verstärkt an dieser Präidee an. Als Biomarker galten zunächst makroskopische Körpersäfte licher Crundlagenforschung (Biophysik, Biochemie, Molekularbiologie), der biomedizinischen Technik und ihren Anknüpfungen an die klinische Praxis und privatwirtschaftlichen Akteur/innen (Gaudillière und Rheinberger, 2004). Das Human Genome Project repräsentiert mit seinem Anspruch auf Vollständigkeit bei der Entschlüsselung des menschlichen Cenoms im Stile der Big Science ein wissenschaftliches Großprojekt, wie er auch im Human Brain Project einen vergleichbaren Ausdruck findet (Mittra, 2016b). 
(vor allem das Blut) oder Gewebeproben, die sich auf ihre mikroskopische biochemische Zusammensetzung untersuchen ließen (Gisler, 2011, S. 142). ${ }^{9}$ Mit der Molekularisierung der Life Sciences allerdings verschob sich die Bedeutung des Biomarkers, der in der Folge nicht mehr aus dem Körper extrahiert wurde, sondern mit bestimmten Strukturen der DNA, sog. Genexpressionen, assoziiert wird. Das Verständnis von Krankheit und Gesundheit verschob sich ebenfalls auf eine vielschichtige, makromolekulare Gemengelage und bewegte sich stetig vom akuten Gesundheitsproblem eines Individuums hin $\mathrm{zu}$ einem latenten Risikospektrum, das sich statistisch auf gesamte Populationen übertragen lässt (Sharon, 2014, S. 114ff.). Auf diese Art und Weise verdrängte ein biotechnologischer Zugriff auf die molekulare Welt den chemischen, der lange Zeit die dominanten Körper- und Krankheitsbilder lieferte.

Die gegenwärtig verwendeten Biomarker stellen Genexpressionen dar, die auf der Grundlage statistischer Wahrscheinlichkeiten als Risiken für bestimmte Krankheitsausprägungen verstanden werden. Insbesondere komplexe Krankheitsbilder wie Krebs, Alzheimer oder Morbus Crohn, bei denen nicht ein monokausaler Ursache-Wirkungs-Schluss gezogen werden kann, sondern ein vielschichtiges Geflecht als Multifaktorenmodell vorliegt, führten wohl überwiegend zum Aufschwung des molekularbiologischen Denkens in der Biomedizin (ebd.). Gesundheitsrisiken können so populationsbasiert errechnet und Risikopatient/innen durch genetische Testverfahren ausgemacht werden, die anschließend für bestimmte Präventionsmaßnahmen gewonnen werden können (ebd., S. 115). Darüber hinaus sind durch die Biotechnologie und Biopharmaka wie monoklonale Antikörper wirksame Interventionen bei einer Vielzahl an Erkrankungen entstanden. Mithilfe der molekulargenetischen Grundlagenforschung versprach man sich, das Gen zu finden, das eine bestimmte Krankheit auslöst. Der Blick direkt in die DNA versprach höchste Objektivität, Naturwissenschaftlichkeit und Glaubwürdigkeit, die weit über die Fähigkeiten der organischen Chemie hinausgingen.

Mit der Molekularisierung der Biomedizin entwickelte sich somit das Körperbild einer Assemblage, d.h. der Körper erscheint in eine Vielzahl von „Betrachtungsebenen aufgelöst“" (Michael, 2011, S. 65). Das molare Körperbild aus Zeiten

Als besonders eindrückliches Beispiel hierfür gilt der handelsübliche Schwangerschaftstest, der im Urin das hCG Hormon (humanes Choriongonadotropin) misst. Der sog. Schnelltest, der bestimmte Stoffe in Blut oder Urin nachweist, gilt für dieses Paradigma als besonders repräsentativ. Ein weiteres Beispiel, bei dem das Schlüssel-Schloss-Prinzip besonders deutlich wird, bildet etwa die Forschung nach Wirkstoffen, mithilfe dessen sich Patient/innen das Rauchen abgewöhnen können: Ausgehend von den Nikotinrezeptoren im Gehirn suchte man nach Stoffen, die diese belegen, sodass Nikotin-Moleküle keinen Anschluss finden und ihre Wirkung nicht entfalten können. Dieser war mit dem sog. Cytisin gefunden (Wehling, 2011, S. 1080). 
des clinical gaze bestand in der Vorstellung eines ganzheitlichen, in sich geschlossenen Systems und wurde durch einen partikulär aufgebauten Körper ersetzt, das sich aus verschiedenen Teilsystemen unterschiedlicher Komplexitätsgrade zusammensetzt (ebd., S. 118). Entsprechend der Präideen von Einheitswissenschaft und Hierarchie der Wissenschaften (vgl. 5.2) lassen sich wissenschaftliche Disziplinen entsprechend ihrer Betrachtungsebene und Probembezug den verschiedenen Auflösungsebenen zuweisen. Interdisziplinäre Zusammenarbeit wird in diesem Sinne als Übersetzung bzw. Extrapolation zwischen den Ebenen verstanden, eine Präidee, die insbesondere in der Arzneimittelentwicklung vorherrscht (vgl. 3.3). Medizinische Forschung, Behandlung und Gesundheitswissenschaften wurden so im Verlauf noch stärker an naturwissenschaftliche Grundlagenkenntnisse gebunden, während naturwissenschaftliche Forschung und klinische Praxis in der Folge immer weiter auseinanderrückten (Egloff et al., 2011). Insbesondere zu den grundlegenden Naturwissenschaften Physik, Biologie und Chemie bestehen in der Praxis kaum noch Anbindungen, obwohl deren Wissensbestände noch immer Teil der Medizinausbildung sind.

Der Graben zwischen diesen epistemischen Welten von Naturwissenschaften und Biomedizin wird in gesundheits- und wissenschaftspolitischen Diskursen verhandelt. Insbesondere die Qualität des Wissenstransfers zwischen bench und bedside (synonym für Grundlagenforschung und klinische Anwendung) wird dabei problematisiert, was das Paradigma der translationalen Medizin verändern möchte (Coller, 2008). Den empirischen Analysen zufolge, die diesem Buch zugrunde liegen, sind diese Bemühungen allerdings erschwert, denn die Übersetzungsschwierigkeiten ergeben sich nicht etwa aus der Natur der Sache, als die sie häufig im Diskurs reifiziert werden. Die molekulare Welt selbst stellt eine boundary category dar, an der sich zahlreiche disziplinäre Kollektive mit unterschiedlichen epistemischen, technischen, sozialen, wirtschaftlichen und politischen Prämissen treffen. Diese befinden sich in Abgrenzung zueinander in der Aushandlung von Ressourcen und eine Übersetzung zwischen den Wissensbeständen ist nicht möglich, sofern diese in Abgrenzung zueinander konstituiert sind. Medizinisches und insbesondere chemisches Wissen sind durch das Verhältnis einer Arbeitsteilung charakterisiert, die nach wie vor im Wissenschaftsverständnis von Ärzt/innen wie Chemiker/innen vorherrscht und sich in dem fast schon instinktiven Verweis molekularer Basiskonzepte wie der Chiralität aus dem Wissensschatz der Medizin zeigt:

Lang: welche Rolle spielt Chiralität in Ihrer Disziplin?

Medizin-Doktorandin Ali-Zadeh: Nein, nicht dass ich wüsste (2) obwohl (1) ja gut, wir machen ja ne Kooperation (1) auch wieder mit den Chemikern, die uns ein Medikament herstellen, das es eigentlich nicht mehr gibt. Und da ist es auch so, dass (1) nicht alle Enantiomere davon biologisch WIRKsam sind. Aber da ER des herstellt 
[lacht] hab ich keine Ahnung, welche da jetzt genau-also von demher spielt es glaube ich schon 'ne Rolle. [...] Also bei Medikamenten aufjeden Fall (1) aber sonst (1) ehrlich gesagt (1) in der klinischen Chemie hab ich's auch NIEMALS mehr gehört (6) Nein, ich glaube ehrlich gesagt, dass es gar nicht SO 'ne große Rolle SPIELT also (3) es is halt (1) mir fällt jetzt wirklich nichts ein wo Chiralität im Alltag wirklich wichtig wäre.

Diese Präidee einer Arbeitsteilung, die sich hier in der Fokussierungsmetapher eines Chemikers, der den klinischen Forschungsprozess als Zulieferer unterstützt, manifestiert, ist in den Narrativen angehender wie ausgebildeter Ärzt/innen in Forschung wie Praxis omnipräsent. Die Annahme, dass medizinische Forschung sich zur naturwissenschaftlichen im Sinne arbeitsteilig getrennter Sphären verhält, dient der Wissensorganisation im Feld. Das Beispiel der Chiralität zeigt dabei, dass Student/innen der Medizin bereits früh ein arbeitsteiliges Denken einüben und festigen, indem sie zu unterscheiden beginnen, welche Aspekte, Theorien, Konzepte, Praktiken zu den eigenen bzw. fremden Wissensbeständen zählen. Diese ein- und ausgrenzende Strategie im Umgang mit fachfremden Wissensinhalten und Praktiken ermöglicht zum einen ein gewisses Maß an Handlungsfähigkeit, denn das Wissen, das hypothetisch medizinisch relevant sein könnte, ist unbegrenzt. Die Grenzarbeit zwischen Medizin und Naturwissenschaften, im Rahmen deren laufend die Grenzen neu festgelegt werden, was Mediziner/innen an naturwissenschaftlichem Basiswissen wissen müssen, dient dabei dem Erhalt einer medizinischen Handlungsfähigkeit. Die Arbeitsteilung und Grenzen der Zuständigkeit werden von medizinischer Seite aus nach Möglichkeit verteidigt um die Handlungsfähigkeit angesichts eines sonst drohenden indefiniten Regresses gewahrt.

Der Modus der Arbeitsteilung, der konstitutiv für das Selbstverständnis der befragten Ärzt/innen ist, impliziert eine geschlossene Abtrennung von den Naturwissenschaften auf epistemischem, praktischem, ästhetischem und normativem Wege. Die normativen Aussagen kreisen stets um die Frage: Was muss man wissen und was nicht? Mediziner/innen verstehen den Kosmos der Medizin als ein in seiner Gänze nicht unüberschaubares Makrogebilde ohne klaren Anfang und Ende, das jedoch ordnungsschaffende Strukturen aufweist. Klare Strukturen bilden etwa die Fachbereiche und Subdisziplinen der klinischen Praxis sowie spezifische Zuständigkeitsbereiche in der Forschung und es herrscht ein ausgeprägtes Hierarchiebewusstsein (Coller, 2008). Praktische Tätigkeiten und Wissensebenen sind funktional differenziert, die Grenzen des jeweiligen Zuständigkeitsbereiches implizit bekannt und werden nach Möglichkeit nicht übertreten. Der Fokus liegt auf der Anwendung extern generierten Wissens, das Menschen auf individueller sowie kollektiver Ebene helfen soll. Den Narrativen der Ärzt/innen zufolge bauen dabei die Wissensebenen von Naturwissenschaften und Medizin konsekutiv aufeinander auf und wenn ein Forschungsprozess die einzelnen Stufen erfolg- 
reich abschreitet, wird der Gesamtprozess erfolgreich sein. Die Chiralität wird ebenfalls ausschließlich im Sinne dieses Prinzips verhandelt. Sie wird als Eigenschaft klar umrissen und einer Disziplin, der generalisierten Chemie, zugeschrieben. Abstraktionen und Übertragungen des Prinzips auf andere wissenschaftliche Fragestellungen als die Arzneimittelentwicklung fallen ihnen erheblich schwerer als den Vertreter/innen anderer Disziplinen, die etwa einen Universalismus der Natur postulieren.

Sämtliche Interview-Partner/innen bewerten die im Medizinstudium vermittelten naturwissenschaftlichen Grundlagen als äußerst rudimentär. Die Vermittlung chemischer Konzepte wie der Chiralität wird als stark vereinfacht und auf unmittelbar medizinbezogene Probleme reduziert wahrgenommen. Daraus speist sich ein hierarchisches Verhältnis, das bei den Medizinstudierenden in Ablehnung und Unwillen gegenüber chemischem Wissen resultiert. Die Qualität chemischer Inhalte im Medizinstudium weicht deutlich von regulären, chemischen Wissens $\mathrm{ab}$, das den Chemiestudent/innen vermittelt wird, sodass niemals eine ebenbürtige Expertise erreicht werden kann und man auf eine spätere Arbeitsteilung bauen muss. Die bereits zitierte Doktorandin drückt es folgendermaßen aus:

Seit Februar mache [ich] jetzt meine Doktorarbeit. Ich mache dafür ein Freisemester um halt einfach in den experimentellen Laboralltag reinzukommen, weil als Mediziner hat man da ja nicht SO VIEL Vorerfahrung außer ein bisschen Biochemie und so Praktika wo du dir Acetat auf den Laborkittel kippst und dann sagst: „Oops, der löst sich auf!"

Diesen Eindruck eines erheblichen Qualitätsgefälles in den fachspezifischen Chemiegrundlagen bestätigen auch meine teilnehmenden Beobachtungen im Chemiepraktikum für Student/innen der Humanmedizin. Die Chiralität als Konzept bzw. Gegenstand gilt dort nur ein weiterer lästiger, chemischer Aspekt, der mit geringer intrinsischer Motivation aufgenommen wird: „[stöhnt] jetzt gibt es da auch noch eine rechte und linke Version von 'nem Molekül, ich kann nicht mehr!", wie es ein Student beklagt. „Es ist ein Graus, das ist so kompliziert, ich weiß“ beschwichtigt ihn der Dozent, ein Doktorand der anorganischen Chemie. Es besteht ein permanenter Aushandlungsprozess um den Stellenwert chemischen Wissens, der auf die molekulare Eigenschaft der Chiralität projiziert wird. Manche Professor/innen und auch einige Studierenden beschweren sich darüber, wie wenig Medizinstudierende tatsächlich über die Naturwissenschaften wissen und dass die gängige Lehrpraxis immer mehr dieser Inhalte herauskürzt (Boeck, 2004). Auf der anderen Seite ist allen Akteur/innen bewusst, wie wenig sich diese Inhalte im späteren Berufsleben als präsent erweisen, mit konstantem Verweis auf besagte Arbeitsteilung.

Hinzu kommt die Feststellung, dass sich sämtliche Akteur/innen darüber bewusst sind, dass die naturwissenschaftlichen Wissensbestände der Mediziner/in- 
nen denjenigen der Fachdisziplinen selbst unterlegen sind. Medizinstudent/innen werden von Chemiker/innen, Physiker/innen und Biolog/innen ausgebildet, die ihnen stark vereinfachte, im Umfang erheblich reduzierte Inhalte vermitteln, die sie mehr auswendig lernen als verstehen müssen (Germer et al., 2002). Wie die Analysen des Lehrmaterials- und Betriebes zeigen, präsentiert sich dabei die Chemie als essentiell für die Medizinausbildung und es wird der Eindruck einer künftigen Omnipräsenz chemischen Wissens im späteren Berufsfeld vermittelt. Die Chemie hält darüber eine Hierarchie gegenüber der Medizin aufrecht und legt fest, welche chemischen Inhalte Teil des Medizinstudiums sind. Chemiker/innen portionieren und teilen das Wissen $\mathrm{zu}$, sie haben den Überblick darüber, wie Mediziner/innen denken und wollen deren Einstellung zur Chemie beeinflussen. Dies geschieht etwa über die Inszenierung chemischer Basiskonzepte wie der Chiralität, die als Brücke zwischen Chemie und medizinischem Fachwissen verhandelt wird. Die Lehre (Vorlesung sowie Praktika) werten die Chiralität für die Medizin auf, es wird der Eindruck einer künftigen Omnipräsenz ${ }^{10}$ und Relevanz vermittelt. Das zitierte Vorwort eines Chemielehrbuches für Medizinstudent/innen enthält die zentralen Narrative, die sich auch in der Interviewstudie und den Beobachtungen im Feld widerspiegeln:

Für viele Schulabgänger bzw. Studienanfänger stellt sich die Chemie als Hexerei dar. Chemiker scheinen neue Stoffe nach denselben geheimnisvollen Prinzipien herzustellen, wie Zauberer Hasen aus dem Zylinder hervorholen. Da sich Studierende der Medizin und Zahnmedizin gleich zu Beginn ihres Studiums mit den naturwissenschaftlichen Fächern auseinandersetzen müssen, weil Kenntnisse der Chemie als Basis für das Erlernen anderer vorklinischer und klinischer Fächer, vor allem der Biochemie, aber auch der Toxikologie und Pharmakologie, unabdingbar sind, führt dies nicht selten zu Frustrationen. Viele Studierende überstehen einen Chemie-[...]kurs nur, indem sie Grundlagen wie mathematische Gleichungen auswendig lernen. Meine Absicht ist es, Sie zu überzeugen dies NICHT zu tun! Verlange Sie von sich selbst, von diesem Buch sowie von ihren Studienbetreuern Erklärungen zum „WAS und WARUM“ in der Chemie. Mit anderen Worten: Wenn Sie die tieferen Ursachen und Zusammenhänge kritisch hinterfragen und dann auch verstanden haben, anstatt alles einfach auswendig zu lernen, werden Sie schnell bemerken, dass Sie sich das Leben und Lernen wesentlich erleichtern (Margaretha, 2002, S. v.).

Die in diesen Narrativen abgewertete epistemische Praxis des Auswendiglernens hat allerdings einen nachhaltigen Lerneffekt: Wie sich in den Interviews mit den

10 In dem beobachteten Laborpraktikum nimmt die Stereochemie einen großen Raum ein (3 von 10 Terminen im Laborpraktikum der Human- und Zahnmedizin sowie 2 von 5 Terminen in der Veterinärmedizin sind chiralen Strukturen gewidmet). 
befragten Ärzt/innen verschiedener Altersstufe offenbart, sind selbst nach vielen Jahren die Lehrbuchdefinitionen zur Chiralität und anderer chemischer Inhalte abrufbar, selbst wenn diese Begriffe im jahrelangen Berufsalltag so gut wie keine Rolle spielen. So wird auch bei dem folgendem Vertreter der Radiologen im Zitat deutlich, dass die Beschäftigung mit den Naturwissenschaften für das Medizinstudium zwar konstitutiv ist, allerdings als extern und außermedizinisches wahrgenommen wird. Die naturwissenschaftlichen Grundlagen machen das Studium schwieriger, dienen aber auch aufgrund von Wartesemesterregelungen als Einstiegshilfe in das Medizinstudium, falls die Studienzulassung nicht direkt möglich ist.

Lang: Was verstehst du denn unter Chiralität?

Tartini: CHIRALITÄT Hände [lacht] da war irgendwas [lacht] vor langer Zeit [lacht] Chemie, oder?

Lang: Ja erzähl mal

Tartini: Na ja, ich mein das ist die Spiegelbildlichkeit, oder? Von Händen, die sind ja spiegelbildlich und sowas gibt's ja auch bei-wir hatten da so'n STECKmodell. Ich hatte ja auch 'nen Chemiegrundkurs also ich mein [viele Leute wissen] gar nicht, dass so $n$ ' Medizinstudium ganz viel beinhaltet, also es fängt an mit Physik des is mir ja dann erlassen worden, weil ich ja vorher Physik hatte. Da wurde mir der Schein anerkannt. Dann Chemie, dann war's Biochemie dann war's also Biochemie, Physiologie, dann auch Psychologie, Anatomie, Histologie also Pathologie und dann eben Rechtsmedizin so in den klinischen Semestern gibt's ja dann auch noch ja dann eben innere Chirurgie Radiologie Strahlentherapie so die kleinen Fächer dann. Jetzt Chiralität, wie g'sagt Spiegelbildlichkeit wir hatten-des hat mich eigentlich schon fasziniert auch wobei Chemie hab ich dann relativ schnell wieder abgelegt [lacht].

Im Gegensatz dazu erweisen sich die praktischen Auseinandersetzungen aus den Chemiepraktika jedoch als völlig vergessen. Es wird deutlich, dass die praxisbezogenen Objektbeziehungen zum Molekularen nicht relevant sind, da diese durch die Wissenshierarchien und Fremdzuweisungen im Feld erschwert bzw. gar nicht aufgebaut werden. Angehende Mediziner/innen lernen nicht, sich mit den Geräten und Stoffen $\mathrm{zu}$ identifizieren. Sie sind sich stets darüber bewusst: „wir machen hier Chemie“. Die Chemie (stellvertretend für die molekulare Welt) wird nicht als Teil der Medizin verstanden, was zu einer Ablehnung führt, denn das eigene Wissen wird aufgrund der Wissenshierarchien und der Arbeitsteilung mit der Chemie ohnehin niemals vollwertig sein. Wie kommt es nun dazu, dass bei ausgebildeten Ärzt/innen und klinischen Forscher/innen die Chiralität in ihrer Definition noch immer derart präsent ist, die Zuständigkeit für den Umgang mit ihr jedoch völlig externalisiert wird? Dies zeigt sich insbesondere in der Auseinandersetzung mit dem historischen Fall des Thalidomids und der Rolle, die die 
molekulare Eigenschaft der Chiralität dabei spielte. Dieser Fall stellt ein Knotenpunkt in der Grenzarbeit zwischen Chemie und Medizin dar, denn es dient als Beispiel für einen Fall, bei dem die etablierte Arbeitsteilung grundlegend und mit dramatischen Folgen versagt hat.

\subsection{Das Versagen der Arbeitsteilung im Contergan-Fall}

Der Contergan-Fall wird zu den tragischsten und schwerwiegendsten Arzneimittel- ${ }^{11}$, Justiz- $^{12}$ und Politikskandalen ${ }^{13}$ des 20 . Jahrhunderts gezählt, denn das teratogene (fruchtschädigende) Thalidomid-Präparat schädigte weltweit etwa 10.000 Neugeborene bzw. tötete viele weitere bereits während der Schwangerschaft. Der Contergan-Fall ${ }^{14}$ markierte eine Zäsur in der öffentlichen Wahrnehmung der Pharmabranche und ging mit einem erheblichen Vertrauensverlust in wissenschaftliche Expertise (Steinmetz, 2003) und erhöhter Risikowahrnehmung einher (Luhmann, 2001). Eine Reform des Arzneimittelgesetzes regulierte im Zuge der Contergan-Katastrophe die Zulassung von Arzneimitteln und brachte für die pharmazeutische Industrie Einschränkungen in der Produktzulassung mit sich (Roth, 2007). Insbesondere in der Bundesrepublik Deutschland, Großbritannien und Kanada spinnt sich seit dem Bekanntwerden der Schäden und dem fast zehn Jahre andauernden Gerichtsverfahren gegen den Pharmaproduzenten Grünenthal seit den 1960er Jahren ein dichter Diskurs (Kirk, 1999). Die Aktivitäten und Stellungnahmen von Betroffenenverbänden, aus dem Gesundheitswesen, Pharmabranche, Elternvereinigungen, Journalist/innen, Politiker/innen und etlichen weiteren Beteiligten wurden bereits ausführlich diskursanalytisch untersucht (Freitag, 2005). Bis heute steht in diesen Diskursen die Schuldfrage im Mittelpunkt; ob und wie die Katastrophe hätte verhindert werden können und wie die Firma Grünenthal sich zu dem Fall verhalten sollte (Roth, 2007).

Auch an den Schnittstellen zwischen chemischen Wissenschaften und Biomedizin hat sich ebenfalls ein Diskurs um den Contergan-Fall etabliert, der allerdings von diesen öffentlichen und juristischen Debatten eher unabhängig geführt wird. Während die Chiralität neben anderen molekularen Eigenschaften des Thalidomids in letzteren kaum Beachtung findet, stellt die Chiralität des Thalidomids einen markanten Grenzstein in der Grenzarbeit zwischen Biomedizin

\footnotetext{
11 (Lenhard-Schramm und Großbölting, 2017)

12 (Dierichs, 1990)

13 (Daemmrich, 2002)

14 Im Diskurs wird der Contergan-Fall auch als „Contergan-Skandal“ (Cemballa, 1993), "Contergan-Affäre“ (Steinmetz, 2003), „Contergan-Tragödie“ (Jungmayr, 2012), „ConterganKatastrophe“ (Zichner, 2005) oder „Contergan-Bombe“ (Schwerin, 2009) bezeichnet.
} 
und Chemie dar. Die Spiegelisomerie des Thalidomid-Moleküls schlägt sich in erheblichem Maße auf das bioaktive Wirkungsspektrum des Stoffes nieder und entscheidet u.a. auch über die fruchtschädigende Eigenschaft des Stoffes (Knoche und Blaschke, 1994). In den Diskursen zwischen Chemie und Medizin steht dabei die Aushandlung von Grenzen der Zuständigkeit im Zentrum und welche Rolle verschiedene Disziplinen im Zusammenhang mit Arzneimittelsicherheit spielen. Es entsteht dabei auf der Seite der Medizin eine Spannung, denn einerseits sind Mediziner/innen, wie im vergangenen Abschnitt angeführt, auf eine gewisse Arbeitsteilung mit Naturwissenschaftler/innen angewiesen, um überhaupt angesichts der sonst ausufernden Wissensbestände handlungsfähig zu bleiben. Andererseits steht der Contergan-Fall für ein Beispiel, bei dem die Arbeitsteilung mit schwerwiegenden Konsequenzen versagt hat, weswegen das Fallbeispiel im Rahmen der Grenzarbeit zwischen Chemie und Medizin immer wieder Gegenstand ist.

Darüber hinaus war auch die Geschichte des Thalidomids als Arzneistoffs mit der Marktrücknahme von Contergan 1961 nicht zu Ende, denn ThalidomidPräparate sind bis heute im Einsatz - allerdings nicht als Schlafmittel für Schwangere, sondern als Lepra-Medikament oder als Chemotherapie bei Leukämie und es werden laufend neue Anwendungsbereiche erschlossen (Kim und Scialli, 2011). Das Wirkungsspektrum des Thalidomids und seiner Derivate sind von der stereochemischen Struktur abhängig und verschiedene Enantiomere können völlig unterschiedlich im Körper metabolisieren und wirken (Bützer, 2009). Mediziner/innen kennen die Chiralität aus diesem Grund vor allem im Zusammenhang mit Contergan, denn in der medizinischen Ausbildung dient das Molekül als paradigmatisches Beispiel für diese sonst eher unscheinbare chemische Eigenschaft und dient zugleich als Legitimation dafür, warum auch angehende Ärzt/innen der Human-, Zahn- und Veterinärmedizin zumindest über chemische Grundkenntnisse verfügen sollten. Die naturwissenschaftlichen Grundlagen genießen im Medizinstudium erstens keine sonderliche Beliebtheit (Barr et al., 2010) und zweitens wird ihre eigentliche Notwendigkeit in bildungspolitischen Diskursen zur Gestaltung und Schwerpunktsetzung des Medizin-Curriculums immer wieder infrage gestellt bzw. verteidigt (Feuerstein, 2011). Es folgt ein historischer Überblick über die beiden Diskursstränge zum Contergan-Fall und welche Rolle sie jeweils bis heute für die Grenzarbeit zwischen Chemie und Biomedizin spielen.

Der Thalidomid-Wirkstoff kam 1957 mit den Arzneimitteln Contergan und Contergan forte ${ }^{15}$ nach aus heutiger Sicht mangelhaften, da nicht rechtlich

15 Andere Handelsnamen lauteten etwa Softenon und Doriden; eine übersichtliche Aufzählung der Produktnamen und Vertriebsländer findet sich bei (Bützer, 2009, S. 5) und (Kirk, 1999, S. 55). 
vorgeschriebenen, toxikologischen Studien in Deutschland, der Schweiz und 44 anderen Ländern als Einschlafhilfe und Medikament gegen Morgenübelkeit für Schwangere auf den Markt (Ridings, 2013, S. 36). Das Medikament wurde von der Herstellerfirma Grünenthal mit viel Werbeaufwand als „völlig harmlos“ vermarktet (Kessel, 2013), insbesondere da bis heute keine letale Dosis nachgewiesen wurde und Suizide selbst bei gleichzeitiger Einnahme von 144 Tabletten nicht möglich sind (Neuhaus und Ibe, 1960). Dass Thalidomid im Gegensatz $\mathrm{zu}$ den damals umstrittenen Barbiturat-Präparaten weder süchtig macht noch Überdosierungen direkt lebensgefährlich sind, wirkte sich ebenfalls positiv auf die Verkaufszahlen aus (Wilsmann und Zwingenberger, 1996, S.66). Entsprechend wurde das Medikament in vielen Ländern zum Bestseller, da die „Ungiftigkeit“ von Arzneimitteln angesichts der besorgniserregenden, tabletteninduzierten Suizidrate positiv bewertet wurde (Kirk, 1999, S. 53). Die Entwicklung von Thalidomid-Präparaten gilt in der Pharmaziegeschichte als paradigmatisches Beispiel für eine magic bullet drug zur Zeit der golden age of drug discovery, einer niedermolekularen, chemischen Verbindung, die in der Entwicklung aufgrund eines spektakulären serendipity moments (vgl. 3.2) nur geringe Kosten verursacht und dank der schnellen Marktreife (aufgrund einer scheinbar günstigen Pharmakovigilanz) zu enormen Umsätzen geführt hat (Ruthenberg, 2016).

1954 soll der Mediziner und Chemiker Heinrich Mückter in seiner Funktion als Laborchef bei der Firma Grünenthal (Standort Stollberg bei Aachen) zusammen mit seinen Kollegen Herbert Keller und Wilhelm Kunz das Molekül Thalidomid erstmalig als Produkt eines glücklichen Zufalls synthetisiert haben (Kunz et al., 1956). Als sie das Molekül an Versuchstieren testeten, stellten sie bei diesen eine sedierende Wirkung fest (Kirk, 1999, S. 52). Klinische Studien, die auf die Tierversuche an Hamstern, Ratten und Mäusen folgten, erbrachten die unterschiedlichsten Ergebnisse in Bezug auf Verträglichkeit und Nebenwirkungen (Ruthenberg, 2016, S. 59). So wurde in der klinischen Pilotstudie an 20 Patienten, denen eine sehr hohe Dosis verabreicht wurde, Verdauungsprobleme, Ohrensausen und Gliederzittern festgestellt (Ridings, 2013, S. 36f.). Andere Ärzt/innen ${ }^{16}$ in Deutschland und der Schweiz (die Grünenthal mit gratis Produktmustern ausgestattet hatte) stellten abweichende Symptome fest bzw. bestätigten die gute Verträglichkeit, da kein hang over-Effekt zu beobachten war, die Leberwerte im normalen Bereich blieben und das Suchtrisiko als äußerst gering bewertet wurde (ebd.). Insgesamt wurde der Thalidomidwirkstoff (vorgeblich) an 20.000 Versuchspersonen getestet, bevor es auf den Markt kam. Aus dieser Population gingen später zehn Kinder mit thalidomidinduzierten Fehlbildungen hervor (Luhmann, 2001, S. 68). 
Als Contergan in Deutschland 1957 bundesweit in sämtlichen Apotheken und medizinischen Einrichtungen rezeptfrei erhältlich war, geizte Chemie Grünenthal nicht mit Werbemaßnahmen, die sich gezielt an Ärzt/innen und Apotheker/innen richteten, um das neuartige Schlafmittel speziell im gynäkologischen und pädiatrischen Kontext als marktführendes Arzneimittel zu etablieren (Ruthenberg, 2016, S. 57). Aufgrund der propagierten „völligen Ungiftigkeit“ avancierte das Medikament schon bald zu „West-Gemany's baby-sitter“ (Taussig, 1962, S. 1109), da viele Eltern es den ärztlichen Empfehlungen entsprechend ihren Kindern und Säuglingen verabreichten (ebd.). Als Contergan gezielt bei Morgenübelkeit in der Schwangerschaft Linderung versprach, wurde es zudem speziell an Schwangere vermarktet und dispensiert. Im Jahr 1961 wurden die höchsten Verkaufszahlen erzielt, als Contergan und Contergan forte monatlich etwa eine Million D-Mark umsetzten. ${ }^{17}$ Der Pharmakonzern Grünenthal verfügte mit den thalidomidhaltigen Präparaten über große Marktanteile und war zugleich in hohem Maße von dessen Erfolg abhängig, da Contergan etwa die Hälfte des jährlichen Gesamtumsatzes des Unternehmens einbrachte (ebd.: 56).

Zugleich kamen bereits um 1960 die ersten medizinischen Auffälligkeiten ans Licht, die mit Contergan in Zusammenhang gebracht wurden. $\mathrm{Zu}$ Beginn des Jahres 1960 wurde insbesondere bei älteren Patient/innen, die die Einschlafhilfe konsumiert hatten, eine neuartige Form der Polyneuritis bemerkt (Frenkel, 1961). Es handelte sich um eine Nervenschädigung der Extremitäten, bei den sich in Händen, Füßen und Beinen Kribbeln sowie motorische Schwierigkeiten bis zum Gefühlsverlust bemerkbar machten (Taussig, 1962, S. 1109). Bis Ende des Jahres 1960 sollen bei der Firma 1.600 Berichte über diese Nebenwirkungen eingegangen sein, bei denen es sich teilweise um ernste Fälle handelte (Sjöström und Nilsson, 1972). Als vermehrt Anfragen besorgter Ärzt/innen bei Grünenthal eingingen, wurden diese zunächst teilweise $\mathrm{zu}$ widerlegen versucht oder ignoriert (Dierichs, 1990, S. 64f.). Darüber hinaus wurden gar Fälle beobachtet, in denen Vetreter/innen der Firma Grünenthal gezielt Patient/innen in Kliniken aufsuchten um mit Geld und kostenlosen Behandlungen deren Schweigen zu erkaufen (ebd., S. 65). Die Firma Grünenthal bemühte sich zudem, Positivberichte über Behandlungserfolge zu verbreiten (Kirk, 1999, S. 63).

In der Folge wurden $\mathrm{ab} 1958$ in der Bundesrepublik und etlichen weiteren Ländern bei Neugeborenen immer häufiger Fälle sog. Phokomelie (Robbengliedrigkeit) beobachtet, bei denen es sich ansonsten um eine äußerst seltene Form von Fehlbildungen der Gliedmaßen (Dysmeliesyndrom) handelt. Diese Fehlbildungen an Gliedmaßen und inneren Organen wurden zudem früh mit Grünenthals Thalidomid-Präparaten in Verbindung gebracht (Taussig, 1962). Dieses Dysmelie-Syndrom trat erst ab 1958 überhaupt in nennenswerter Zahl auf (genau 
9 Monate nach Markteinführung) und binnen der vierjährigen Verkaufszeit kamen weltweit etwa 10.000 Kinder mit thalidomidinduzierten, körperlichen Schwerstbehinderungen zur Welt (Enns et al., 2009). Bis zum Sommer 1957 häuften sich die Anfragen aus dem In- und Ausland von Ärzt/innen, Apotheker/innen und Forscher/innen derart, dass Grünenthal die vermeidende Taktik, die es bis dato an den Tag gelegt hatte, änderte. Wie im späteren Gerichtsverfahren deutlich wurde, lagen Grünenthal bis zum Jahre 1960 etwa 1000 solcher Anfragen vor, die jedoch bewusst geheim gehalten wurden (Freitag, 2005, S. 34).

Widukind Lenz, ein Hamburger Kinderarzt, wurde aufgrund seiner eigenen Beobachtungen in der Praxis sowie einem Gespräch mit den Kollegen Wilhelm Kosenow und Rudolf Pfeiffer der Zusammenhang zwischen den Phokomelie-Fällen und Contergan-Einnahmen von werdenden Müttern bewusst (Lenz, 1988). Zunächst zögerlich brachte er diesen Verdacht, der sich in Gesprächen mit Müttern und weitere Kolleg/innen verdichtete, an die Öffentlichkeit, zunächst jedoch ohne den Produktnamen zu nennen (Daemmrich, 2002, S. 144). Fast zeitgleich veröffentlichte der australische Mediziner William McBride einen Artikel, der dieselbe Hypothese äußerte, jedoch war er in der Produktnennung deutlicher (McBride, 1961). Am 26. November 1961 wurde schließlich der Verdacht, dass die gehäuften Phokomeliefälle mit dem Arzneimittel Contergan in Verbindung stünden, in der Welt am Sonntag unter dem Titel „Mißgeburten durch Tabletten? Alarmierender Verdacht eines Arztes gegen ein weitverbreitetes Medikament" publik gemacht (Welt am Sonntag, 1961). Auch wenn hier Contergan nicht namentlich genannt wurde, schien doch allen klar zu sein, von welchem Medikament die Rede war, denn noch am selben Tage erfolgte die Marktrücknahme (Kirk, 1999, S. 83ff.).

Der anschließende, fast zehn Jahre währende Gerichtsprozess gegen die Herstellerfirma Grünenthal stellt ein eigenes Feld dar (Schütze, 1997), das hier nicht in Gänze rekapituliert werden soll. Als wichtiges Fazit lässt sich jedoch in wissenssoziologischer Perspektive feststellen, wie unerbittlich in diesem Prozess und der medialen Repräsentation und Inszenierung über die Bedeutung wissenschaftlicher Beweise gestritten wurde (Steinmetz, 2003). Der Begriff des wissenschaftlichen Beweises und die Aussagekraft wissenschaftlicher Studien wurden in der öffentlichen Wahrnehmung nachhaltig erschüttert. Fakten und Tatsachen, Behauptungen, Hypothesen und Vermutungen zum teratogenen Mechanismus des Thalidomid-Moleküls wurden darüber hinaus je nach Interessenslage umgedeutet, interpretiert, abgelehnt oder angenommen (Knightley et al., 1979). In den Diskussionen und Gerichtsakten schlugen sich Deutungskämpfe vor dem Hintergrund der Wahrung finanzieller Interessen vonseiten Grünenthals und Opfervertreter/innen nieder (Gemballa, 1993, S. 161ff.). Darüber hinaus waren Ansehen und Glaubwürdigkeit der pharmazeutischen Industrie ebenso bedroht (Sjöström und Nilsson, 1972) wie das der politischen Vertreter/innen (insbesondere das Gesundheitsministerium Elisabeth Schwarzhaupts) (Steinmetz, 2003, S. 210). 
Zudem ist an dieser Stelle anzumerken, dass von Anfang an versucht wurde, den Kreis der offiziell Geschädigten möglichst klein zu halten, indem die Behinderungen vor allem an Fehlbildungen der Gliedmaßen festgemacht wurden und andere, wie etwa bestimmte Fehlbildungen der inneren Organe, aus der Definition der "Contergan-Geschädigten“ ausgeklammert wurden (Freitag, 2005, S. 54ff.). Der Prozess wurde im Dezember 1970 mit einem Freispruch wegen geringer Schuld abgeschlossen. Auch wenn die Firma Grünenthal den Prozess fast bist zur Verjährung herausgezögert hatte, sich wenig kooperativ gezeigt hatte, Akten vernichtet und wissenschaftliche Ergebnisse zu relativieren versucht hatte, wurde ihr dennoch zugute gehalten, nicht wesentlich anders gehandelt $\mathrm{zu}$ haben als es zur damaligen Zeit in der pharmazeutischen Industrie Standard gewesen war und nicht gegen geltendes Recht verstoßen zu haben (Kirk, 1999, S. 96). Mit der Einrichtung einer Stiftung für die Contergan-Geschädigten mit einem Stiftungsvolumen von 100 Millionen D-Mark schien Grünenthal ein großes Opfer zu bringen. Doch bis heute wird kontrovers über Entschädigungsmaßahmen diskutiert und weitere Forderungen reißen angesichts der immensen Langzeitpflegeund Therapiekosten der heute ca. 2400 teils schwerstbehinderten contergangeschädigten Menschen nicht ab (Enns et al., 2009).

Der Cotergan-Fall ging als erster gravierender Arzneimittelskandal in die Geschichte ein und prägte die junge Bundesrepublik Deutschland nachhaltig (Schwerin, 2009). Das Vertrauen der Menschen in die Wissenschaft und deren Fortschritt schwand und das Verhältnis von Wissenschaft, Politik und Gesellschaft erlebte einen Wandel (Luhmann, 2001, S. 76ff.). Die Ausmaße der Contergan-Katastrophe spielen im kollektiven Gedächtnis eine erhebliche Rolle, etwa in Gestalt der charakteristischen körperlichen Merkmale der sog. Contergan-Kinder (Freitag, 2005). Darüber hinaus hat der Contergan-Fall das Gefühl der Ohnmacht in der Öffentlichkeit angesichts einer Verquickung zwischen profitgierigen Pharmariesen, einer unfähigen Justiz und politischer Vertreter/innen, die viel zu spät eingreifen, um die Bevölkerung zu schützen (Steinmetz, 2003). Die Schuldfrage ist in den öffentlichen und politischen Debatten bis heute präsent. Ärzt/innen wie Widukind Lenz oder Frances Kelsey, die als Verantwortliche der US-amerikanischen Food and Drug Administration (FDA) die Marktzulassung von thalidomidhaltigen Präparaten in den USA trotz allen Widerstandes verzögern konnte, gelten als Held/innen im Diskurs (Daemmrich, 2002).

Während die Aufarbeitung des Contergan-Falls in der Öffentlichkeit sich überwiegend mit dieser Schuldfrage beschäftigte und mit der Frage, wie mit den Tausenden, teils schwerbehinderten Kindern umzugehen sei, beschäftigten sich Chemiker/innen, Pharmakolog/innen und klinische Forscher/innen mit den molekularen Mechanismen der Teratogenität und wie solche Fälle in Zukunft zu vermeiden seien. Als direkte Folge des Contergan-Falles wurde das ArzneimittelGesetz von 1961 (das ursprünglich nur die Registrierung neuer Arzneimittel vor- 
sah und Pharmaka wie Contergan, die bereits zuvor auf dem Markt waren, gar nicht berührte) 1976 vollständig neu formuliert (Stapel, 1988). Neue Vorschriften zur Pharmakovigilanz erforderten zudem flächendeckend neue labortechnische und experimentelle Methoden, sodass diese in Auseinandersetzung mit dem Contergan-Fall einen Innovationsschub erlebten. Zunächst erfolgte zwischen 1961 und 1968 eine Reihe toxikologischer Studien ${ }^{18}$ an verschiedenen Tiergattungen, die die teratogene Wirkung in verschiedenen Spezies nachzuweisen sowie besonders sensible Schwangerschaftstage und Dosierungen zu ermitteln versuchten (Fabro und Smith, 1966; Helm, 1966; Schumacher et al., 1968).

Zur selben Zeit wurde in diesen Werken auch zunehmend ein Bezug zur Stereochemie und den Spezifika der beiden Stereoisomere des Thalidomids gezogen (Shealy et al., 1965; Fabro et al., 1965). Es wurde festgestellt, dass sowohl das Racemat als auch die beiden Isomere fruchtschädigend wirken, sich für die S(-)-Form des Thalidomids allerdings ein höherer Grad an Toxizität beobachten lässt als für die R(+)-Form (Fabro et al., 1967). Auch die chemischen, pharmazeutischen und toxikologischen Fachdiskurse kreisten um die Frage, ob und wie der Contargan-Fall hätte verhindert werden können. Die Stereochemie und ihre Rolle in der Arzneimittelwirkung steht dabei häufig im Zentrum. In den 1950er Jahren war die chirale Eigenschaft vieler Arzneistoff-Kandidaten und wie dieser Aspekt sich auf dessen Wirkungsspektrum auswirkt zwar bekannt ${ }^{19}$, entsprechende Pharmakovigilanztests war allerdings weder in den Arzneimittelgesetzen vorgeschrieben, noch gängige Praxis der Arzneimittelentwicklung (Roth, 2007). Zumal in den 1960er Jahren die Aufspaltung von racemischen Gemischen in technischer Hinsicht äußerst aufwendig galt, wurde die Berücksichtigung der Stereochemie erst mit Aufkommen leistungsfähiger Flüssig-Chromatografen zur Racematspaltung in den späten 1970er Jahren systematisch berücksichtigt (De Camp, 1989, S. 2).

18 Siehe zur historischen Studienlage die Arbeiten von (Knightley et al., 1979, 255-274) und (Freitag, 2005, S. 30-44). Letztere fasst insbesondere auch die zahlreichen pädiatrischen und orthopädischen Untersuchungen zum Phänomen der Phokomelie zusammen und wie in diesem wissenschaftlichen Diskurs durch Ein- und Ausschlusskriterien das sog. Dysmelie-Kind hervorgebracht wurde.

19 Der Zeitraum zwischen den $1880 e r$ und den 1930er Jahren wird in der Chemiegeschichte als frühe Hochphase des pharmakologischen Enantiomervergleiches eingestuft (vgl. 2.3). Etliche Naturstoffe, deren Derivate und synthetische Stoffe wurden auf ihrer optische Aktivität hin überprüft und dementsprechend in Bezug auf ihre Metabolisierung und Wirkungsweisen in vivo wie in vitro hin erforscht (Gal, 2006, S. 18). Arthur Robertson Cushny legte 1926 einem umfassenden Report über diese Studien vor (Cushny, 1926), bei dem er jedoch das Augenmerk eher auf physikalische Eigenschaften und die optische Rotation der Enantiomere legte als die chemischen Strukturformeln, welche er weitestgehend aussparte (Parascandola, 2012). 
Die Legitimation dieser (relativ teuren) Laborausstattung in der organischen Chemie (vgl. Abschnitt 2.3) begründete sich in den 1970er Jahren über die Verheerungen des Contergan-Falls, der an einer mangelhaften technischen Ausstattung der Labore festgemacht wurde. Für die pharmazeutische Produktentwicklung ebenso wie für die Arzneimittelsicherheit stellte sich in der Folge die Frage, ob ein reines Enantiomer (ohne den „Ballast“ bzw. die „Verunreinigung" eines wirkungslosen zweiten Isomers) nicht die Wirkung eines Arzneimittel potentieren bzw. bei einem schädlichen Enantiomer das Risiko verringern ließe (Ariëns, 1984). Mit weiteren Reformen internationaler Arzneimittelgesetzgebungen zog schließlich die Chiralität Ende der 1980er Jahre in die Vorschriften der Zulassungsverfahren von Pharmazeutika ein (De Camp, 1989). Dies fiel mit mehreren historischen Tendenzen zusammen, die in Kapitel 2 beschrieben werden: dem Ende der golden age of drug discovery und dem Beginn der sog. Innovationskrise in der Pharmaindustrie, wobei die Chiralität als zusätzliche, kostspielige Hürde im Zulassungsverfahren und als Innovationshemmnis abgelehnt wurde. Die Chiralität sorgt seither im F\&E-Prozess industrieller Arzneimittel für ein erhöhtes Maß an Komplexität, zumal im Rahmen der Pharmakokinetik chiraler Moleküle es dazu kommen kann, dass einzelne Isomere unter bestimmten Bedingungen im Körper in die jeweils andere Form racemisieren, sprich umwandeln können (Meierhofer et al., 2001). Darüber hinaus ermöglichten die neuen Technologien der Racematspaltung auf der anderen Seite der organisch-chemischen Grundlagenforschung einen regelrechten Forschungsboom und die Stereochemie erfreute sich nicht zuletzt ihrer pharmazeutischen Anwendungen großen Zulauf ehe der Trend nach der Jahrtausendwende wieder abebbte, als das Feld als ausgeschöpft galt (vgl. 3.2).

Lang: Wie erklären Sie sich, dass [die Stereochemie in den 1990er ]ahren] diese Attraktion hatte?

Prof. Pejačević: Damals hat unser Lehrstuhlinhaber ganze BÜCHER drüber geschrieben und das war natürlich (.) von so fundamentaler Bedeutung, dass es ja irgendwie jeden Menschen fasziniert. Thalido-Thalidomid war nicht SO lange her muss ich sagen. Das ist ja fast in meiner GeneraTION in der das passiert ist und dann kam eben die ganzen Verfahren aufzur stereoselektiven Synthese, zur chiralen Chromatografie zu der Verwendung in der pharmazeutischen Industrie. Und eben die ganze Arzneimittelindustrie (.) hat dann danach auch die Standards verSCHÄRFT wirklich für die Testung von Enantiomeren und auch für den Metabolismus. [...] Ich bin da keine Expertin weil ich halt weder Pharmazeut noch Organiker bin aber ich weiß mit Sicherheit, dass das natürlich ein Kanonenschlag war! Danach ist man ja wirklich erst auf das Problem [der Chiralität] aufmerksam geworden durch dieses Thalidomid. 
Thalidomidhaltige Präparate waren darüber hinaus nie ganz aus der medizinischen Praxis verschwunden. Bereits 1965, nur vier Jahre nach dem Verkaufsstopp der Grünenthal-Präparate, verschaffte ein Arzt in Israel mit einem übriggebliebenen Arzneimittel auf Thali-domid-Basis einem Lepra-Patienten Linderung. Als der Patient daraufhin nicht nur von einem erholsamen Schlaf berichtete, sondern auch ein deutlicher Rückgang der Lepra-Sympto-me beobachtet werden konnte (Sheskin, 1965), fand das Molekül wieder auf den Markt zurück, allerdings unter verschärften Abgaberegelungen. Thalidomid verfügt über ein sehr vielfältiges Wirkungsspektrum, so wirkt es nicht nur sedativ und teratogen, sondern auch antiinflammatorisch wie im Falle von Lepra und antiangiogenetisch, d.h. es verhindert die Bildung einer Blutzufuhr bei Tumoren wie dem multiplen Myelom, einer Form des Hautkrebses (Teo et al., 2005; Wu et al., 2005). Das Monitoring nach der Zulassung thalidomidhaltiger Präparate bzw. strukturverwandter Derivate fällt heute besonders streng aus, insbesondere bezüglich der Prävention bei Schwangeren (ebd.) Die Auseinandersetzung mit der Chiralität in Pharmazeutik, Biomedizin und Stereochemie war in diesem Sinne zwischen den späten 1970er Jahren und der Jahrtausendwende nachhaltig durch die Suche nach den Ursachen für die Contergan-Katastrophe geprägt.

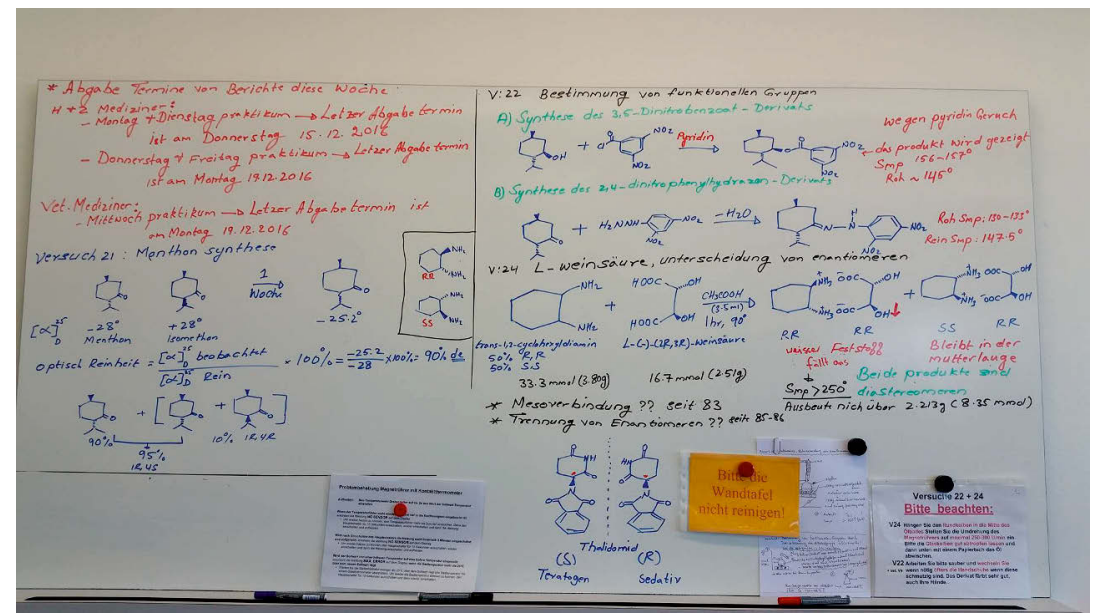

Diese Fotografie (SL) entstand im Rahmen der teilnehmenden Beobachtungen in den Chemiepraktika für Medizinstudent/innen. Die Tafel zeigt den Versuchsaufbau zu stereochemischen Experimenten, die die Student/innen in einer bestimmten Lehreinheit absolvieren müssen. Thalidomid wird dabei besonders hervorgehoben um die Bedeutung der Chiralität in der medizinischen Praxis zu betonen.

In diesem Zusammenhang wurde wiederholt die implizite, etablierte Arbeitsteilung zwischen Medizin und Naturwissenschaften problematisiert und kriti- 
siert, dass innerhalb der biomedizinischen und pharmazeutischen Produktentwicklung zu wenig Kommunikation und Austausch von Wissen zwischen Disziplinen und Feldern vorherrscht (Luhmann, 2001, S. 71ff.). Der Contergan-Fall wurde in der Folge immer wieder als legitimierendes Beispiel für die Forderung nach einem intensiveren interdisziplinären Austausch zwischen Medizin und Naturwissenschaften herangezogen, indem die Verheerungen hervorgehoben wurden, die sog. Übersetzungslücken mit sich führen können. In der Ausbildung angehender Ärzt/innen werden diese regelmäßig mit der Bedeutung chemischer Inhalte im ärztlichen Wissensschatz konfrontiert (vgl. Abb. 5.1). Debatten um den Contergan-Fall kommen im Diskurs i.d.R. dann auf, wenn (vor allem im Zuge der Deakademisierungstendenzen der Medizinausbildung) die Bedeutung chemischer Grundlagenkenntnisse im Medizinstudium infrage gestellt werden. ${ }^{20}$ Insbesondere dann, wenn Zweifel an den chemischen Inhalten im Medizinstudium geäußert werden, funktioniert der Verweis auf den Contergan-Fall als intelligible Rechtfertigung.

Der Verweis auf das Versagen der etablierten Arbeitsteilung im Contergan-Fall dient den Feldern der Chemie dazu, ihren Platz im Gefüge der Wissenschaften $\mathrm{zu}$ behaupten, den sie insbesondere dadurch bedroht sieht, dass lebenswissenschaftliche Zugriffe auf die molekulare Welt zunehmend dominant werden und diejenigen der organischen Strukturchemie (vor allem vor dem Hintergrund des Paradigmenwechsels vom kleinen zum großen Molekül in der Pharmaindustrie, vgl. Abschnitt 4.1) als nicht mehr zeitgemäß erscheinen lassen. Heute gilt die Chiralität in den Feldern der Medizin (in Bezug auf Arzneistoffe, etwa in der Pharmakologie) als beherrschbar und man geht im Allgemeinen davon aus, dass sich eine Katastrophe wie der Contergan-Fall nicht wiederholen wird. Ärzt/innen vertrauen voll auf Fertigpharmazeutika und ihre eigenen Fähigkeiten in der Verschreibung.

20 Die Debatten um den Status der Medizin und der Mediziner/innen-Ausbildung schwanken zwischen der Idee, die Medizin im Gefüge der Naturwissenschaften zu halten und auf der idealtypischen Gegenseite Ärzt/innen zu „Gesundheitsingenieur/innen“ und aus der akademischen Landschaft zu lösen, da es sich ohnehin um keine vollwertige Wissenschaft handelt (Biller-Andorno and Roduit, 2016; Feuerstein, 2011). Psychiater Dr. Silcher schätzt die Situation folgendermaßen ein: „Also die Medizin ist ja an und für sich eine sehr pragmatische Wissenschaft. Ich hatte mal als Studentenvertreter eine Diskussion mit einem Professor, der meinte damals, man müsste eigentlich die Medizin aus der Akademie RAUSnehmen. Also man müsste wieder einen Schritt zurück machen und der Mediziner soll wie ganz am Anfang zu den Metzgern gehen, also so eine LEHRE machen. Weil 99 Prozent der Mediziner interessieren sich nicht für Wissenschaft, sondern für die SACHE und für das Handling von Problemen. Also zwischen eine SAU zu schlachten oder äh richtig zu schlachten und ein Teil von einem Menschen richtig chirurgisch zu entfernen, ist die Nähe (1) eher gegeben als zum Beispiel zwischen einem Chirurgen und einem Psychiater". 
Auch wenn die Chiralität in der pharmazeutischen Pharmakovigilanzforschung heute der allgemeinen Wahrnehmung nach abschließend erforscht ist und von enantiospezifischen Wirkungsspektren keine negativen Überraschungen mehr erwartet werden, spielt die Chiralität noch immer eine wichtige Rolle im Verhältnis zwischen Chemie und Biomedizin. Die Abgrenzungsdynamiken der beiden Felder treten darin deutlich zutage. Auf der einen Seite lassen sich über die Chiralität (insbesondere des Thalidomid-Moleküls) Brücken schlagen zwischen organischer Chemie und der medizinischen Praxis. Die Relevanz chemischer Inhalte lässt sich ebenso plausibel vermitteln wie die des interdisziplinären Austausches in Zeiten sich hybridisierender Felder und zuspitzender Expertisen. Auf der anderen Seite werden chemische Grundlagen in der Medizinausbildung zunehmend rechtfertigungsbedürftig. Dies zeigt sich auf drei Ebenen: der erwähnten Deakademisierung der ärztlichen Ausbildung in Anbetracht überfüllter Curricula, den ausgelagerten klinischen Laboranalysen, die kaum ein/e Ärzt/in noch selbst anfertigt und dem Trend, dass sich die medizinische Forschung zunehmend in Richtung hybrider, molekularer Lebenswissenschaften bewegt. Organische Chemie und Biomedizin driften in Anbetracht dieser Tendenzen zunehmend auseinander, was insbesondere für die Felder der Chemie ein Abhandenkommen wichtiger etablierter Legitimationsnarrative in der Akquise akademischer Ressourcen bedeutet.

\subsection{Reflexionen zum Konzept der translationalen Medizin}

Die Felder von Chemie und Biomedizin befinden sich in einem historisch gewachsenen, fluiden Abgrenzungsverhältnis, das permanent ausgehandelt und aktualisiert wird. Dieses Abgrenzungsverhältnis im Sinne einer Arbeitsteilung spiegelt sich auch in den verschiedenen wissenschafts- und forschungspolitischen Trends sowie Innovationsdispositiven des 20. und 21. Jahrhunderts wieder. Da die etablierten Grenzen zwischen Medizin und Naturwissenschaften mit der einheitswissenschaftlichen Präidee einer prinzipiellen Unterscheidung zwischen Grundlagen- und Anwendungsforschung korrespondierten, wurde die Arbeitsteilung zwischen diesen Feldern in der Forschungspolitk des Kalten Krieges auch institutionell verankert (Schauz, 2014a). Dass die Naturwissenschaften die grundlegenden Naturgesetze erforschen, aus denen die Industrie Anwendungen entwickelt, die dann von Mediziner/innen ans Patient/innen-Bett transferiert werden, war lange als Innovationsmodell fest in den Fachidentitäten der beteiligten Kollektive verankert. Ärzt/innen sind sich bis heute bewusst darüber, "reine Anwender" $\mathrm{zu}$ sein, die in eigener Forschung und Praxis auf externe „Zuweiser" von Wissensbeständen, Methoden, Molekülen und Produkten angewiesen sind. Die Präidee der Arbeitsteilung ermöglicht in einem komplexen 
Feld ohne klare Grenzen zumindest vage Orientierungspunkte für die Grenzen der eigenen Zuständigkeit. Im Translationsparadigma, das mittlerweile in den Innovationsstrategien zahlreicher anwendungsorientierter biotechnologischer und ingenieurswissenschaftlicher Bereiche etabliert ist, wird die Präidee einer Arbeitsteilung allerdings in jüngster Zeit problematisiert und erfährt eine Disruption (Mittra, 2016c).

Im Zusammenhang mit forschungspolitischen Diskursen um Innovation, Interdisziplinarität, Großprojektforschung und einer verstärkten Zusammenarbeit von Akademie und Privatwirtschaft hat sich in den medizinischen Fachwissenschaften, der internationalen Wissenschaftspolitik sowie den Innovationsdispositiven verschiedener gesundheitsbezogener Industriezweige das Programm der translationalen Medizin etabliert (Westfall et al., 2007). Das forschungspolitische Programm der translational medicine, deren Entwicklung und Implementierung zur Mitte der 1990er Jahre ihren Lauf nahmen, übersetzt gewissermaßen die Innovationsdispositive des weiter gefassten translational turn ${ }^{21}$ in die Felder der Biomedizin (Chai und Shih, 2013). Ausgangspunkt des neuen Paradigmas bildet die Diagnose langwieriger und wenig produktiver Innovationsprozesse der pharmazeutischen und medizintechnologischen Industrie (Mittra, 2016c). Diese werden erstens auf Kommunikationsprobleme aufgrund $\mathrm{zu}$ geringer interdisziplinärer Ausrichtung (Ioannidis, 2006) und zweitens auf eine datenbasierte Komplexitätssteigerung ${ }^{22}$ zurückgeführt, die den Innovationsprozess technisch behindert (Folkers, 2011). So wird davon ausgegangen, dass insbesondere der enorme Wissenszuwachs in den Molecular Life Sciences zu einer Diffusion der Wissensformen und einer Zuspitzung von Expertisen führt, die an den behandlungspraktischen Medizinbetrieb kaum noch anschlussfähig sind (Woolf, 2008).

Das Programm der translationalen Medizin wurde in der Folge entworfen, um die diagnostizierten Übersetzungslücken zwischen Grundlagenforschung und

21 Ein translational turn lässt sich darüber hinaus in verschiedenen anderen Disziplinen feststellen, vor allem in den Ingenieurs- und Technikwissenschaften. Auch dort besteht Übersetzungsbedarf, da anwendungsbezogene Produktentwicklung und Innovation kaum noch an die abstrakten physikalischen Erkenntnisse der letzten Jahre anknüpfen können (Chai und Shih, 2013, S. 3).

22 Wie Bernadette Bensaude-Vincent kritisiert, werden Komplexität, Unsicherheit und nach wie vor eher enttäuschende Erfolgsraten pharmazeutischer Innovation nicht einfach durch eine Erhöhung der Rechenleistung gelöst, denn die Übersetzungsschwierigkeiten ergeben sich aus der molekularen, biotechnologischen Perspektive selbst. Die komplexen biopharmakologischen Zusammenhänge lassen sich nicht exklusiv auf das (weit verbreitete) Credo der Reduzierbarkeit pharmakologischer Wirkung auf einen zugrundeliegenden genetischen Code lösen (Bensaude-Vincent, 2007). Vielmehr präsentieren sich die in den Lebenswissenschaften und ihre Innovationsprozesse involvierten Kollektive und Disziplinen als äußerst heterogen, was Methoden, Theorien und Prämissen betrifft. 
Anwendungen zu schließen, die sich zwischen verschiedenen Kollektiven der Biomedizin (und auch nach außen) aufgetan haben (Mittra, 2016a). In diesem Abschnitt wird erläutert, welche Konsequenzen sich aus dieser Programmatik der Translation für die Grenzarbeit der chemischen und biomedizinischen Felder ergeben: Die Chemie als monolithische Systemwissenschaft gilt entsprechend der Präideen des Translationsparadigmas als veraltet, innovationsfeindlich und konservativ, sodass sie sich mittlerweile von der Realität medizinischer Anwendungen weit entfernt bewegt (ebd.). Durch die gezielte Förderung hybrider, interdisziplinärer, problembezogener wissenschaftlicher Felder - vor allem im Bereich der Molecular Life Sciences und Biotechnologie - wird an diesem Beispiel besonders deutlich, wie die Chemie allmählich an epistemischem Einfluss auf die Medizin verliert. Der Chemie zugerechnete Konzepte wie die Chiralität verschwinden aus der Produktentwicklung und geraten auch in der Praxis zunehmend in Vergessenheit. Dies zeigt sich wiederum in paradigmatischer Art und Weise in den Zugriffen auf die molekulare Welt, bei der hybride Lebenswissenschaften entsprechend der Innovationsmodelle der klassischen organischen Synthesechemie als überlegen gelten. ${ }^{23}$

In der Folge werden die bisherige Entwicklung, Programmatik und die Präideen der translationalen Medizin erörtert und vor dem Hintergrund der Befunde zur Boundary Work zwischen Chemie und Biomedizin neu bewertet. Diese Lesart erlaubt Rückschlüsse über die Lage der Chemie in Zeiten sich hybridisierender Felder und sich wandelnder Innovationsdispositive. Die Chemie steht dabei stellvertretend für die als klassische Großdisziplinen verstandenen Felder, die in Anbetracht gegenwärtiger forschungspolitischer Dispositive als Auslaufmodell gelten. Darüber hinaus erfolgt eine kritische Revision der Diagnose der Übersetzungsprobleme zwischen den erwähnten Kollektiven, die nicht ausschließlich einer wachsenden Komplexität wissenschaftlicher Wissensproduktion und Datenfülle geschuldet sind. Vielmehr wird argumentiert, dass diese aus der inhärenten Grenzarbeit zwischen Medizin und Chemie hervorgehen, die sich bereits zu Zeiten der frühen akademischen Etablierung der Chemie ergeben hat und in die zeitgenössischen epistemischen Kulturen eingeschrieben sind und damit trotz aller Probleme essentiell für die Stabilität des Feldes.

Bei der translationalen Medizin handelt es sich um kein einheitliches Konzept, vielmehr versammelt sich unter dem Begriff ein vielstimmiger Diskurs mit

23 Die vorliegenden Analysen zum Paradigma der translationalen Medizin und die entsprechenden Thesen zu den Widersprüchen der Programmatik gehen auf meine unveröffentlichte Masterarbeit (Lang, 2014) zurück. Diese bestehenden Befunde werden vor dem Hintergrund der vorliegenden Analysen zur Grenzarbeit zwischen Chemie und Medizin neu gelesen und kontextualisiert, sodass hiermit ein Originalbeitrag vorliegt, der weit über die Befunde der Masterarbeit hinausgeht. Die entsprechende Stelle wird im Text kenntlich gemacht. 
teilweise widersprüchlichen und gegenläufigen Ansätzen, Präideen und politischen Zielsetzungen. Als kleinster gemeinsamer Nenner besteht allerdings der Anspruch, den Wissenstransfer zwischen verschiedenen Instanzen des Medizinbetriebes $\mathrm{zu}$ erleichtern und die Wissensübersetzung $\mathrm{zu}$ beschleunigen (Mittra, 2016c, S. 87). Häufig fällt in diesem Zusammenhang das Schlagwort „from bench to bedside", was sich auf die Forderung bezieht, dass Labor- und Grundlagenforschung stärker in Austausch mit der klinischen Forschung und Praxis gestellt werden sollen, da diese sich im Zuge der Ausdifferenzierung des biomedizinischen Betriebes mehr und mehr voneinander entfernt haben (Woolf, 2008, S. 211). Translationale Mediziner/innen sind dem Ideal nach Personen, die in mehreren Bereichen qualifiziert sind und etwa auf molekularer Ebene mit Hilfe von Biomarkern oder Tiermodellen maßgeschneiderte Diagnosen entwickeln und aus diesen direkt Anwendungen an das Krankenbett übersetzen können. Zu diesem Zweck werden spezielle Studiengänge ${ }^{24}$ ins Leben gerufen, außerdem Journals gegründet, Konferenzen abgehalten, Koordinierungsstellen eingerichtet und Preise verliehen (Vignola-Gagné et al., 2013).

Die translationale Medizin ${ }^{25}$ wurde in den späten 1990er Jahren in den USA als Teil der nationalen Gesundheitsstrategie des National Institute of Health gefördert (Zerhouni, 2005). Das Programm steht für eine stärkere Vernetzung von klinischer Grundlagenforschung und Praxis ein, was in der Phrase "from bench to bedside" Ausdruck findet. Mit Translation ist dabei allerdings eher in seltenen Fällen eine Übersetzung im linguistischen oder semantisch-qualitativen Sinne, etwa zwischen Fachkulturen oder bestimmten Formen des tacit knowledge gemeint (Coller, 2008), sondern zwischen sog. Auflösungsebenen, also zwischen submolekularen, molekularen, makromolekularen, biomolekularen, zellspezifischen und patient/innen-bezogenen Datensätzen. Das Translationsparadigma knüpft damit an eine Wissenschaftstheorie des Emergentismus an (Folkers, 2011): Wie in den

24 Spezielle Ausbildungs- und Studienprofile im Sinne der translational medicine wurden etwa in Masterstudiengängen wie Master of Science in Translational Medical Research oder der Molekularen Medizin ausgegründet. Expert/innen, die in diesen interdisziplinären Lebenswissenschaften ausgebildet werden, sollen die bisher eingesetzten Ärzt/innen ersetzen, die qua Ausbildung zu weit von der klinischen Forschung entfernt sind und zudem in Anbetracht der Krisen der Cesundheitssysteme ohnehin mehr in der Patient/innen-Versorgung benötigt werden (Fulda, 2012).

25 In denselben Zeitraum fällt zudem die Entstehung und Förderung weiterer Medizinstile und Konzepte, wie etwa Evidenzbasierter Medizin, Individualisierter Medizin, Personalisierter Medizin oder auch der Stratifizierten Medizin, deren Überschneidungen fließend sind und keine klare Abgrenzung erlauben (Kollek, 2012). Ein wesentlicher Unterschied zwischen translationaler und personalisierter Medizin liegt darin, dass biomarkerbasierte Diagnosen und Behandlungen im Rahmen der Individualisierten Medizin beim zu behandelnden Individuum enden und in der translationalen Medizin darüber hinaus Erkenntnisse formuliert werden sollen, die sich wiederum auf eine gesamte Population übertragen lassen (ebd.). 
Abschnitten 6.2 und 2.3 deutlich wird, war insbesondere die pharmazeutische Industrie eine Fürsprecherin neuerer Emergenztheorien, die sich von der Entwicklung neuer computertechnologischer Verfahren der künstlichen Intelligenz eine Antwort auf ihre Innovationskrise erhoffte. Mit dem Paradigma der translationalen Forschung war für verschiedene Industriezweige mit Fokus auf den Gesundheitsbereich eine plausible Erklärung für die Innovationskrise gefunden, die trotz technischem Fortschritt und systematischer Umstellung auf biotechnologische Verfahren kein Ende zu nehmen schien: Übersetzungsprobleme zwischen Wissensbeständen wurden als Ursache definiert, politisiert und für Förderprogrammatiken operationalisiert. Emergenztheorien bilden gewissermaßen ein wissenschaftsphilosophisches Fundament des Translationsparadigmas, zumal beide ein Weltbild implizieren, das von einer in Seinsschichten und Komplexitätsniveaus klassifizierten Wirklichkeit ausgeht, zwischen denen Wissen übersetzt werden muss - was allerdings aufgrund von ansteigender Datenkomplexität erschwert wird (Weber, 2013).

In Anbetracht dieser Prämissen wird auch der forschungspolitische Kontext der neuen Bio Healtheconomy deutlich, die sich im Zuge der fundamentalen Paradigmenwechsel in der Forschungspolitik seit den 1980er Jahren herausgebildet hat (Mittra, 2016c, S. 87). Eine Innovationspolitik, die verstärkt auf public-private partnerships, Interdisziplinarität, problem- und gegenstandsorientierte sowie anwendungsorientierte Programme setzte, war in der praktischen Umsetzung verstärkt mit Fragen und Problemen der Zusammenarbeit und Wissenskommunikation in immer größeren und heterogeneren Kollektiven ${ }^{26}$ und Verbünden konfrontiert (ebd.). Diese Fragen und Probleme wurden in der Ausrichtung der translationalen Programmatik erstens auf Biomarker und zweitens auf die Frage nach der Extrapolation von Forschungsdaten adressiert:

Komplexe Krankheitsbilder wie Krebs, Morbus Alzheimer oder auch Diabetes werden wie in Kapitel 4 ausführlich behandelt, in ihrer Erforschung, Diagnostik, Behandlung und Prävention immer weniger alleinig der niedermolekularen Chemotherapie des small molecules-Paradigmas und den entsprechenden Biorezeptoren überlassen. Vielmehr herrschen im Zuge der diskutierten BioMolekularisierung der Medizin Körper- und Krankheitsbilder vor, die an biochemische, molekularbiologische und biotechnologische Wissensbestände und Technologien anknüpfen. In Diagnostik und Behandlung spielen nun nicht mehr Krankheitserreger, die den Körper von extern befallen eine Rolle, sondern vor allem biochemische Stoffe des eigenen Körpers auf Molekülebene und in

26 So ist es kein Zufall, dass translationale Programme häufig inform institutionenübergreifender Forschungscluster implementiert werden, die Unispitäler, Hochschulen, medizintechnische und pharmazeutische Privatfirmen miteinander verknüpfen, die sehr hohe Investitionen erfordern. 
welcher Weise sie etwa mit Bestandteilen der DNA interagieren (Egloff et al., 2011). Der Denkstil wandelte sich also von der Annahme, körperfremde Stoffe würden den Körper schädigen ${ }^{27}$, dahingehend, dass Krankheitswahrscheinlichkeiten bereits in der Genetik des Körpers festgelegt seien, mithilfe von Gentests prognostizierbar und vor allem durch maßgeschneiderte biotechnologische Therapeutika behandelbar wären. Die Idee wandelte sich somit auch hin zur Annahme einer grundsätzlichen Pathologie des Gewebes, das durch Prävention verhindert werden kann (Gisler, 2011).

Im Zuge dieser Trends erfuhren statistische Verfahren (vor allem in ihrer automatisierten Form) eine erhebliche Aufwertung in den Feldern der Biomedizin, die im Translationsparadigma zum zentralen Medium der Wissensübersetzung avancierten. Die Extrapolation zwischen verschiedenen Datensätzen spielte dabei insbesondere in den Industriezweigen der Pharmazeutik und Medizintechnologie eine erhebliche Rolle. Die lange Dauer und Risiken der biomedizinischen Produktentwicklung ergeben sich demnach nicht nur aus den verschärften $\mathrm{Zu}$ lassungsverfahren, sondern auch daraus, dass Forschungsergebnisse sich häufig nicht von einer auf eine andere Betrachtungsebene extrapolieren ließen (Folkers, 2011). Zwischen einer spezifischen Genexpression (Genotyp) und einem konkreten, makroskopischen körperlichen Erscheinungsbild (Phänotyp) kann es etwa zu einer extrem großen Varianz an Phänomenen kommen. Ein weiteres Problem sind verschiedene Tiermodelle und ihre begrenzte Übertragbarkeit auf menschliche Populationen (Martić-Kehl und Schibli, 2012) oder auch die gängige Praxis der klinischen Studien, Behandlungen im ersten Schritt an gesunden, männlichen Freiwilligen $\mathrm{zu}$ testen, was bei einer heterogenen Kohorte multimorbider Patient/innen verschiedenen Alters und Geschlechts völlig andere Ergebnisse hervorbringt (und letztere gar gefährden kann).

Der formulierte Übersetzungsbedarf zwischen Grundlagenforschung und Anwendungen am Behandlungsbett ergibt sich nicht zuletzt daraus, dass die Lücke zwischen beiden Bereiche angesichts wachsender Komplexität an Forschungsdaten schwer überbrückbar sind. Dennoch legitimiert sich der Ruf nach Translation über als statistisch signifikante kommunizierte Erfolge und unverzichtbare biotechnologische Innovationen, die über Leben und Tod entscheiden (vgl. Abb. 5.2). Im Zentrum dieser Narrative steht die Diagnose, dass sich eine unproduktive Ausdifferenzierung, metaphorisch als Graben umschrieben, zwischen sog. Grundlagenforschung und den medizinischen Anwendungsbereichen herausgebildet hat

27 Das Verhältnis von Pathologischem und Gesundem hat sich soweit gewandelt, dass nicht wie früher von einem normalen Zustand der Cesundheit ausgegangen wird (anhand dessen konstruierten Ideals dann Krankheit als Abweichung verstanden wird), sondern: „da alle Menschen bestimmte Dispositionen für Krankheiten in sich tragen, gibt es auch kein »risikofreies «Cenom mehr; in diesem Sinne werden genetische Risiken normal“ (Kollek, 2012, S. 43). 


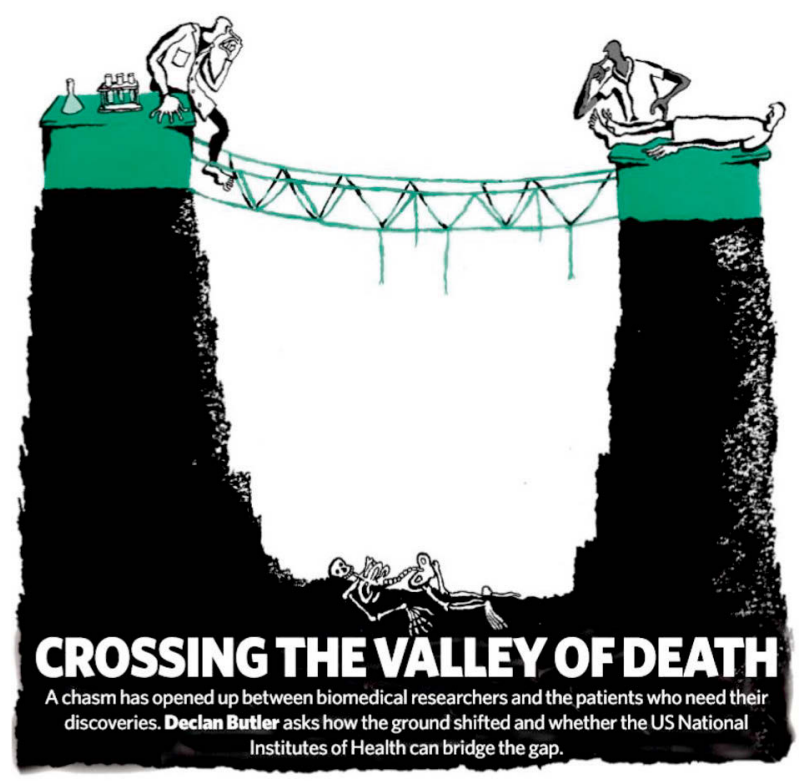

Die prominenteste Legitimationsstrategie des Translationsparadigmas bezieht sich in der Medizin auf das Narrativ personalisierten Nutzens maßgeschneiderter Behandlungen im Dienste von Patient/innen. Übernommen aus (Butler, 2008).

(Butler, 2008). Demzufolge wird es im Medizinbetrieb immer schwieriger, den enormen Wissenszuwachs im molekularen Wissen über den menschlichen Organismus und seine Erkrankungen in konkrete und erfolgreiche Gesundheitsanwendungen und Behandlungserfolge zu überführen. Diese Diskurse weisen viele Überschneidungen mit den Debatten um die sog. Innovationskrise der pharmazeutischen Industrie (vgl. Abschnitt 4.1) auf, denn auch die Industriezweige der Biotechnologie, Medizintechnik und Public Health-Strategien hängen i.d.R. diesem Innovationsmodell an (Vignola-Gagné et al., 2013).

Neuere Auseinandersetzungen und Evaluationen $\mathrm{zu}$ den bisherigen Auswirkungen der Translationsprogrammatik deuten allerdings darauf hin, dass die bisherigen Maßnahmen die bestehenden Kommunikationsgräben zwischen Disziplinen und Instanzen der biomedizinischen Produktentwicklung zu einer paradoxen Situation geführt haben: So weiten sich im Zuge translationaler Maßnahmen die „research silos" bzw. Gräben zwischen den Kollektiven eher noch aus anstatt diese zu überbrücken (Lang, 2014). So stößt man in der Literatur (vgl. Abb. 5.3) häufig auf die sog. translationalen Blöcke mit den Bezeichnungen T1, T2, T3 und $\mathrm{T}_{4}$ (Khoury et al., 2007, S. 665). Es handelt sich dabei um größere Kollektive von Fachwissenschaftler/innen und entsprechenden exoterischen Kreisen in 


\begin{tabular}{|c|c|c|c|}
\hline $\begin{array}{l}\text { Translation } \\
\text { research phase }\end{array}$ & Notation & Types of research & Examples \\
\hline TI & $\begin{array}{l}\text { Discovery to candidate health } \\
\text { application }\end{array}$ & $\begin{array}{l}\text { Phases I and II clinical trials; observational } \\
\text { studies }\end{array}$ & $\begin{array}{l}\text { Is there an association between } B R C A \\
\text { mutations and breast cancer? }\end{array}$ \\
\hline $\mathrm{T} 2$ & $\begin{array}{l}\text { Health application to evidence-based } \\
\text { practice guidelines }\end{array}$ & $\begin{array}{l}\text { Phase III clinical trials; observational } \\
\text { studies; evidence synthesis and } \\
\text { guidelines development }\end{array}$ & $\begin{array}{l}\text { What is the positive predictive value } \\
\text { of } B R C A \text { mutations in at-risk } \\
\text { women? }\end{array}$ \\
\hline $\mathrm{T} 3$ & Practice guidelines to health practice & $\begin{array}{l}\text { Dissemination research; implementation } \\
\text { research; diffusion research Phase IV } \\
\text { clinical trials }\end{array}$ & $\begin{array}{l}\text { What proportion of women who meet } \\
\text { the family history criteria are tested } \\
\text { for } B R C A \text { and what are the barriers } \\
\text { to testing? }\end{array}$ \\
\hline T4 & Practice to population health impact & $\begin{array}{l}\text { Outcomes research (includes many } \\
\text { disciplines); population monitoring of } \\
\text { morbidity, mortality, benefits, and risks }\end{array}$ & $\begin{array}{l}\text { Does } B R C A \text { testing in asymptomatic } \\
\text { women reduce breast cancer } \\
\text { incidence or improve outcomes? }\end{array}$ \\
\hline
\end{tabular}

Die pluralen Konzeptionen der translationalen Medizin, die mittlerweile nebeneinander bestehen, wurden einem Versuch der Kategorisierung unterzogen. Insgesamt vier verschiedene Subkonzepte versuchen, einzelne Übersetzungsschritte im biomedizinischen Innovationsprozess zu steuern. Entnommen aus (Khoury et al., 2007, S. 666).

Forschungspolitik und Privatwirtschaft, die jeweils ein anderes Verständnis von translationaler Medizin teilen. Wie in Fußnote 23 besprochen, beziehen sich die folgenden vier Absätze auf die Ergebnisse meiner unveröffentlichten Masterarbeit aus dem Jahre 2014 (Lang, 2014), die hiermit zum ersten Mal publiziert werden. Die Ausdifferenzierung des forschungspolitischen Konzepts in die translationalen Blöcken T1-4 sowie die Analyseergebnisse ihrer jeweiligen Charakteristika, Bedeutungszusammenhänge und Präideen stellen das zentrale Thema der Masterarbeit dar. Die zentrale Aussage der Masterarbeit - dass sich die translationale Medizin selbst ausdifferenziert hat und paradoxerweise der Übersetzung bedarf anstatt den Übersetzungsbedarf zwischen medizinischen Kollektiven zu gewährleisten wird in der Folge ebenfalls referiert und in Anschluss an die Forschungsfrage nach der Grenzarbeit der Chemie neu gelesen.

„T1 translational research“ verspricht, biomolekulare Krankheitsmechanismen auf der Grundlage von Biomarkern zu verstehen und daraus geeignete Diagnosen, Therapien und Medikamente zu entwickeln (Woolf, 2008, S. 211). Es umfasst die Phasen I und $\mathrm{II}^{28}$ der etablierten klinischen Testreihen (Szilagyi, 2009, S. 74), die darauf abzielen, quantitative Häufigkeiten, Wahrscheinlichkeiten und Zusammenhänge zu erheben. Ti versteht sich als die Grundlagenforschung im translationalen Gesamtprogramm und findet in starker Anlehnung an das $\mathrm{Hu}$ man Genome Project auf der molekularbiologischen Ebene statt: Es geht darum,

28 Bei der Medikamentenentwicklung in Phase I klinischen Studien werden Wirkstoffe und Prototypen von Medikamenten an gesunden Proband/innen angewandt und deren körperliche Reaktionen untersucht (wie sich der Wirkstoff im Körper verteilt, welche Stoffwechselprozesse, Ablagerungen, etc. zu beobachten sind). Wird keine Schädigung der standardisiert gesunden Proband/innen festgestellt, werden auch entsprechend erkrankte Personen miteinbezogen. In Phase II wird experimentell versucht, die optimale Dosierung zu bestimmen (Schumacher und Schulgen, 2008, S. 292ff.). 
so viele Biomarker wie möglich $\mathrm{zu}$ entdecken, zu mappen und in Hinblick auf Wechselwirkungen untereinander zu untersuchen (Grimshaw et al., 2012, S. 2). Aufgrund dieser hohen Konformität mit den Vorstellungen von Translation seitens aktueller research policies, stellt $\mathrm{T} 1$ auch das hegemoniale Modell der translationalen Medizin dar, denn Forschungsvorhaben im Sinne dieser Programmatik erhalten mit Abstand die höchsten finanziellen Zuschüsse und andere Ressourcen des wissenschaftlichen Feldes (ebd.).

$\mathrm{T} 2$ hat sich in Abgrenzung zu T1 herausgebildet und ist vor allem durch die Kritik an diesem definiert, die primär auf der Beobachtung fußt, dass die Innovationen der Molekulargenomik und -Technologie nicht wirklich bei den Patient/innen ankommen. Ihre Forschungstätigkeiten finden primär in klinischen Studien der Phase III $^{29}$ statt und beschäftigt sich mit phänotypischen Ausprägungen von Krankheitsformen (Khoury et al., 2007). In ihrer epistemischen Ausrichtung forscht die T2 translationale Forschung in die entgegengesetzte Richtung: Anwendungen werden auf der Grundlage empirisch beobachteter Patient/innen-Daten erhoben. Sie basieren damit im Gegensatz zu Ti nicht auf Rückschlüssen vom Genotyp (basalen molekularen Grundlagen des Lebens), sondern auf dem Phänotyp (Szilagyi, 2009, S. 74)). Neben diesen "major translational blocks" sind noch zwei weitere Konzepte zu erwähnen, $\mathrm{T} 33$ und $\mathrm{T} 4$, die primär im Bereich des Public Health Managements angesiedelt sind und weder der Grundlagenforschung noch der klinischen Forschung zuzurechnen sind. Im Sinner einer Übersetzung "from benchside to bedside to curbside" (Mata und Davis, 2012) sind diese auf die Implementierung der in $\mathrm{T} 1$ und $\mathrm{T} 2$ entwickelten Innovationen wie Immuntherapien und diagnostischer Testverfahren in der klinischen Praxis als maßgeschneiderte, individualisierte Behandlungskonzepte für bestimmte Risikogruppen zugeschnitten (ebd).

Mit dieser Ausdifferenzierung in vier translationale Blöcke trat die paradoxe Situation ein, dass sich verschiedene Forschungskollektive genau innerhalb der institutionellen Gräben angesiedelt haben, die sie eigentlich zu überbrücken versprechen (Lang, 2014, S. 39). Die translationale Medizin kämpft in ihrem Etablierungsprozess gegen festgefahrene Strukturen, Präideen und hegemoniale Denkstile des biomedizinischen Betriebes an: Die Arbeitsteilung zwischen verschiedenen Disziplinen und Sphären (Labor, Klinik, Büro) der medizinischen Wissensproduktion (Szilagyi 2009: 71). Die Tendenz, dass sich mit der Ausdifferenzierung dieser Disziplinen und Expertisen auch das Wissen in immer kleiner werdenden Kollektiven konzentriert, sodass Wissensübersetzungen langwierig und

29 Bei klinischen Studien der Phase III handelt es sich um langwierige Wirksamkeitsuntersuchungen mit großen Patient/innenpopulationen, die Wirksamkeit, Unbedenklichkeit bei möglichst verschiedenen Gruppen von Patient/innen nachweisen sollen (Schumacher und Schulgen, 2008, S. 17). 
voller Kommunikationsprobleme sind, stehen ihr im Weg. Die Kritik an immer enger gefassten Expertisen ist in den aktuellen biomedizinischen Diskursen omnipräsent (Clarke und Shim, 2011, S. 180ff.). Die translationale Medizin setzt sich auf ambivalente Weise mit diesem Befund auseinander, da sie zum einen die Verhältnisse kritisiert, aus denen sie selbst erwachsen ist und die dem eigenen Handeln zugrunde liegt. Letzten Endes reproduzieren und verstärken Programmatiken der Translation disziplinäre Ausdifferenzierungen, indem sie nicht bei den bestehenden Disziplinen ansetzen und deren Wissenstransfer verbessern, sondern wiederum neue, hybride Felder erschaffen und fördern, zwischen denen es wiederum zu übersetzen gilt (Lang, 2014, S. 40.).

In der ambivalenten Auseinandersetzung mit der Präidee der interdisziplinären Arbeitsteilung werden verschiedene Konsequenzen der Hybridisierung biomedizinischer Felder und ihrem Verhältnis zu den Nachbardisziplinen deutlich: Während etablierte Systemwissenschaften und die spezifizierte Arbeitsteilung (insbesondere zur organischen Chemie) zwischen diesen immer mehr infrage gestellt wird, profitieren interdisziplinäre und problembezogene Großforschungsverbände und neu entstandene Technosciences von den shifts in der Forschungspolitik. Ärzt/innen und Chemiker/innen werden zugunsten spezialisierter Lebenswissenschaftler/innen aus der medizinischen Wissensproduktion herausgedrängt. Analog zu den Entwicklungen in der pharmazeutischen Industrie, die sich im Zuge der merger mania der 1990er Jahre weitestgehend von der chemischen Industrie getrennt und biotechnologischen Life Sciences zugewandt hat, verliert auch die klassische organische Synthesechemie zunehmend an Bedeutung in der medizinischen Grundlagenforschung wie auch in der klinischen Praxis aus denen die hauseigene Analytik fast vollständig ausgelagert wurde. Hier zeigt sich in besonderem Maße der Niedergang des small molecule-Paradigmas, wie im vorangegangenen Kapitel ausführlich dargelegt wurde. Auch wenn die organische Chemie nach wie vor die primäre Sozialisationsinstanz zahlreicher externer Disziplinen darstellt, erscheinen ihre Theorien, Bildsprachen und Methoden angesichts der biotechnologischen Dominanz auf einer intuitiven Ebene als nicht mehr zeitgemäß.

Diese Verschiebung der etablierten Feldgrenzen, Arbeitsteilungen und Zuständigkeiten von Disziplinen erfahren seit der molekularbiologischen und biotechnologischen Revolution seit den 1970er Jahren Jahre eine erhebliche Disruption. Zahlreiche hybride, problem- und anwendungsorientierte Disziplinen der Molecular Life Sciences sind entstanden und konkurrieren seither mit Systemwissenschaften wie der organischen Chemie um Ressourcen im Wissenschaftssystem. Es zeigt sich, dass sich die Anwendungsbereiche der Chemie, in denen die Chemie bislang ihre „Nützlichkeit“ (Breslow, 1997) in besonderem Maße legitimieren konnte, immer stärker in die Bereiche Minen- und Bauwesen, fossile Ressourcengewinnung und Batterieforschung verlagern, während die Anwendungsmöglichkeiten 
chemischen Wissens im Gesundheitsbereich tendenziell immer stärker den Biowissenschaften zugerechnet werden (Schummer, 2017c). Damit kommt der Chemie zunehmend die wichtigste Legitimationsquelle abhanden: ihr Nutzen für die menschliche Gesundheit und verschiebt sich in Bereiche, die der menschlichen Gesundheit (und der Umwelt) eher schaden, was sich zudem auf chemophobe Ressentiments in verschiedenen gesellschaftlichen Kontexten niederschlägt.

Die zunehmende Aneignung der molekularen Welt durch die Lebenswissenschaften hat zudem Konsequenzen für den biomedizinischen Wissenstransfer und lässt sich in Zusammenhang mit der paradoxen Situation des Translationsparadigmas setzen: So zeigt sich, dass die häufig kritisierte Ausdifferenzierung von Feldern und Expertisen im biomedizinischen Betrieb, die als Ursache der Probleme des Wissenstransfers bewertet wird, sich als Konsequenz eines inhärenten Abgrenzungsprozesses verstehen lässt. In der sozialen Arena der molekularen Welt ist eine Wissensübersetzung nur bedingt vorgesehen und möglich, denn diese Welt ist umkämpft und selbst als Produkt der disziplinären Grenzarbeit zwischen verschiedenen disziplinären Kollektiven hervorgebracht worden. Die Welt des Molekularen präsentiert sich nicht als einheitliche Betrachtungsebene der Natur, sondern ist selbst abhängig von disziplinär organisierten technischen Zugriffen, ästhetischen wie ikonografischen Traditionen, Fachsprachen und Bezügen. Die Arena des Molekularen ist umkämpft, denn sie wird von konkurrierenden Denkkollektiven bewirtschaftet: mit den Anwendungen im pharmazeutischen, biotechnologischen, materialwissenschaftlichen und datenwissenschaftlichen Hybrid-Disziplinen bietet die molekulare Welt seit der molekularbiologischen Revolution der 1970er Jahre eine schier unerschöpflich anmutende Quelle für kommerziell erfolgreiche Innovationen. Mit dem translational turn kam es gewissermaßen in verschiedenen Wissenschafts- und Forschungsbereichen zu einer Abwertung der etablierten Großdisziplin, die als innovationsfeindlich problematisiert wird. Am Beispiel der Chemie zeigt sich daher, welche Konsequenzen die disziplinären Re-Konfigurationen für die Großdisziplinen im Allgemeinen haben. 
